\title{
An Improved RANSAC based on the Scale Variation Homogeneity ${ }^{1}$
}

\author{
Yue Wang ${ }^{1,2}$, Jin Zheng ${ }^{1,3}$, Qi-Zhi Xu ${ }^{1}$, Bo Li $^{1}$, Hai-Miao $\mathrm{Hu}^{1}$ \\ 1. Beijing Key Laboratory of Digital Media, School of Computer Science and \\ Engineering, Beihang University, Beijing 100191, China \\ 2. School of Computer, Shenyang Aerospace University, Liaoning 110136, China \\ 3. State Key Laboratory of Virtual Reality Technology and Systems, Beihang University, \\ Beijing 100191, China
}

\begin{abstract}
For random sample consensus (RANSAC) method, high levels of contamination may slow down its convergence and impede the acquisition of the optimal model. In this paper, an improved RANSAC algorithm based on SIFT matches is proposed, which could provide robust performance in high levels of contamination and with low computational complexity. The improved RANSAC algorithm is particularly tailored to planar homography, by appropriately exploring feature scales. Firstly, a new feature scale constraint, i.e., the scale variation homogeneity (SVH), is proposed by exploiting the fact that the feature scale ratio of a correct match is approximate to the actual scale variation of two matched images. As a result, the potential correct and false matches can be effectively determined by SVH. Secondly, each model is scored by the number of partial identified false matches instead of all correct matches, which will speed up the evaluation process. Finally, fast converge can be obtained by adaptive selection of the test set for determining termination criterion according to the levels of contamination. Experimental results have demonstrated that the proposed SVH-RANSAC algorithm can perform very well even in the scenario of $90 \%$ contamination level. The number of iterations could be decreased by at least $35 \%$ and computational complexity for the evaluation procedure could be decreased by at least $2.5 \%$ compared with the $\mathrm{LO}^{+}$-RANSAC.
\end{abstract}

Index-terms Scale variation homogeneity; scale ratio; RANSAC; SIFT matches

\section{Introduction}

Any two images of the same planar surface can be related by a homography, where a two-dimensional model can be constructed. Homography estimation is the algorithm to estimate the optimal model parameters based on the input matches. In practice, the input matches are inevitably

\footnotetext{
1 This work was partially supported by the National 863 Program (Project No. 2014AA015104), the National Science Foundation of China (No. 61370124) and the National Science Fund for Distinguished Young Scholars (No. 61125206).
} 
contaminated by false matches due to noises, similar textures, parallax and low contrast. Therefore, proper removal of the false matches is a vital procedure for homography estimation. Ultimately, the optimal model can be estimated.

Scale-invariant feature transform (SIFT) [1] and random sample consensus (RANSAC) [2] have been extensively applied in various computer vision tasks, such as image registration, mosaicking, object recognition and so on. In these and many other applications, the requirements on the robustness in high contamination levels and fast the computational speed are demanding. Therefore, continuous efforts have been devoted to improve RANSAC based on SIFT matches. As a matter of fact, RANSAC is an iterative approach to eliminate the false matches and utilize the correct matches to compute the optimal parameters of a mathematical model. In each iteration, three steps, namely hypothesis generation, hypothesis evaluation and termination criterion test, are carried out. Depending on the complexity of the model, e.g. the size of random samples, the conventional RANSAC can provide reasonable performance at $50 \%$ contamination level, which is commonly assumed to be a practical limit for RANSAC in the sense of robust statistics [3]. However, extensive experimental results have demonstrated that RANSAC is unstable when the contamination level reaches 50 or more in percentage. Therefore, additional efforts should be incorporated in the above-mentioned steps to minimize the influence of high contamination levels.

Hypothesis Generation. For this step, a sample, which represents a subset of matches and contains the necessary non-collinear matches, is selected; and an optimal model is calculated. For purpose of homography estimation, four matches are required to preserve the changes of viewpoint, while three matches are required for the purpose of rotational, translational and zooming parameter estimation. Generally, two sampling strategies could be employed. One is the random selection strategy [2, 4-6], which is not advocated for its large computational complexity, and the other is the strategy of avoiding selection of false matches. In the latter strategy, assisted measures are employed to identify potential false matches. For example, in progressive sample consensus (PROSAC) [7], the matches with lower texture similarity are more likely to be false. However, the texture of a SIFT feature is susceptible to the change of viewpoint and illumination. In BaySAC \& SimSAC [8] and BetaSAC [9], the false matches are identified according to the verification of the historical samples. However, inaccurate historical samples would lead to wrong identification results. More recently, researchers have devoted to impose various scale constraints, and potential false matches could be identified with abnormal scale 
difference [10-14]. This assertion, however, only holds for those matches with similar scales. In this sense, it is only effective in the case that all the matched features contain similar scale, where it becomes ineffective if the scale dramatically changes or the large parallax occurs. In summary, these existing approaches are not robust to identify the potential false matches.

Hypothesis Evaluation. For this step, a model with a maximal number of correct matches within a location error threshold will be scored the highest, while those models with low scores will be rejected. For the original evaluation strategy, all the matches participate in the evaluation [2]. In more recent reported work, the evaluation procedure is only carried out on a subset of matches [3, 15] for computational convenience. However, the evaluation results will be unreliable if the contamination level of the subset is high. Therefore, the acquisition of low level contamination subset is vital for obtaining reliable evaluation performance. Moreover, the threshold of location error could be set in an adaptive manner [16-18] rather than using a pre-defined one.

Termination Criterion. In practice, it is more desirable to have less number of iterations for computational convenience; however, this would prevent the method from obtaining the optimal model. In order to achieve a desirable trade-off between number of iterations and the optimality of the model, maximality is proposed in [7], where the probability of finding a better model after a number of iterations is constrained to be smaller than a pre-defined value. If the levels of contamination become high, however, there remain a number of unnecessary iterations. Non-randomness is another approach [7], which describes the minimal number of correct matches. As long as the number of the acquired correct matches is smaller than a predefined threshold, the model will be rejected even though it is correct. Besides, in order to deal with high level of contamination, GroupSAC [19] tests termination criterion only on the matches locating in the overlapped part of two images, but predicting the overlapped part accurately remains to be a challenging task. Although above-mentioned work has practical limitations, they provide insightful inspiration for our work, such as the constraints of the maximum number of iterations and the minimal number of correct matches.

This paper considers the scale variation homogeneity, where improvements have been incorporated into RANSAC to increase its robustness in high contamination levels and with fast computational speed. More specifically, the robustness measure is defined as the capability of quickly returning the correct matches regardless of the contamination levels. To achieve the robustness, scale variation homogeneity (SVH) is proposed by imposing a new scale constraint, where a correct match should be 
approximate to the actual scale variation of the two matched image. The SVH is not sensitive to the changes of viewpoint and illumination, zoom in-out, and rotation, which has been demonstrated to outperform other sampling constraints. Based on SVH, the potential correct and false matches can be distinguished even if the contamination levels become high. Remarkably, RANSAC could provide advantages in the following three aspects. Firstly, the samples are preferably selected from the set of potential correct matches, which could guarantee the uncontaminated samples to be correctly selected as early as possible. Secondly, the number of partial identified false matches is used to score each model, instead of the number of all correct matches. Due to this particular strategy, the workload of evaluation will be desirably decreased, while the reliable evaluation results can be still preserved. Thirdly, the test set of termination criterion can be adaptively selected according to the contamination level, since the influencing factor of convergence rate could be considered as the contamination levels of the test set. As a result, the algorithm can be terminated in time.

The rest of the paper is organized as follows. In Section II, the concept of scale variation homogeneity, and the method of separating the potential correct and false matches are introduced. In Section III, we describe the proposed SVH_RANSAC in detail. Performance comparison of the SVH_RANSAC to the state-of-the-art is given in Section IV. Finally, conclusion and future work are presented in Section V.

\section{Scale variation homogeneity}

The existing scale constraints are implemented by assuming that the scales of the two matched features are similar. However, this assumption does not always hold, since zoom or parallax would make the scales of two corresponding features become different. In this paper, the scale variation homogeneity, a new scale constraint, is proposed. This approach exploits the property that the scale ratio of correct matches is approximate to the actual scale variation of the two matched image. In other words, the matches whose scale ratios are far away from the actual scale variation would indicate incorrectness. Based on the SVH, the potential correct and false matches can be well separated regardless of the similarity between the scales of the two matched features.

\section{A. Observation of the Scale Ratio Restriction}

The scale of a SIFT feature is determined by the size of the blob-like structure [14]. Thereafter, the scales of the same blob in two images would become different once zoom or parallax occurred in the overlapped images. More specifically, the parallax effect is the difference in the position of an object 
viewed from two different lines of sight, which can be measured by angle of inclination [10]. However, the scale ratio of a correct match should be consistent with the actual scale variation of the two matched images. This scale constraint is denoted as scale variation homogeneity (SVH), which is defined as

$$
S R=s c l_{i} / s c l_{j}
$$

where $s c l_{i}$ and $s c l_{j}$ are the scales of two matched features from $\mathrm{i}$ and $\mathrm{j}$, respectively.

Since there are complex transformation, and simple transformation containing rotating and zooming, the performance of SVH can be extensively observed in the following matching experiments. In these experiments, the reference image is the "graffiti" image as shown in Fig. 1 (a) [5], whose size is $800 \times 640$ in pixel. Target images are obtained by transforming the reference image into the required formats. In each observation, SIFT features are detected with the code provided by Rob Hess [21]. The matching algorithm is chosen to be the k-nearest neighbor $(\mathrm{KNN})$ algorithm with $\mathrm{K}=2$ [22]. Notably, all the acquired matches are sorted by their $S R$ in ascendant order.

Observation A (Rotation): Six target images are generated via rotating the reference image by six different angles $\left(15^{\circ}, 45^{\circ}, 90^{\circ}, 135^{\circ}, 180^{\circ}\right.$ and $\left.315^{\circ}\right)$. The $S R$ distributions of the six cases are presented in Fig. 2. Since there is no zoom in any image pairs, scale variation for the two matched images does not exist. Therefore, the scale variation of each image pair should be equal to 1.0 and the average $S R$ $(\overline{S R})$ of correct matches in each image rotation modes should be equal to 1.0. To justify this argument, the correct and false matches are initially identified in a subjective manner for all the matches obtained by KNN. Each match will then be identified one by one. According to their scale variations, the $S R$ distribution can be divided into three contiguous intervals, $(0, \overline{S R}-\Delta),[\overline{S R}-\Delta, \overline{S R}+\Delta]$ and $(\overline{S R}+\Delta, \infty)$. In interval $[\overline{S R}-\Delta, \overline{S R}+\Delta]$, the corresponding matches are considered to be completely correct, while the corresponding matches can be either correct or false in intervals $(0, \overline{S R}-\Delta)$ and 
$(\overline{S R}+\Delta, \infty)$. In this demonstration, we find that all the false matches identified subjectively are distributed in intervals $(0,0.95)$ and $(1.05, \infty)$. Thus, $\Delta=0.05$ and $\overline{S R}$ is approximately equal to 1 , which is consistent with our argument earlier.

Additionally, the re-projection error is computed to validate the above assertion, where derivation details are given in Appendix A. The re-projection error is an objective geometric error estimation method, and is usually used to evaluate the actual geometric error. In particular, the average re-projection errors for six rotation modes are calculated. Generally, lower average re-projection error indicates more correct matches. Since the matches include both the correct and false ones in interval $(0, \overline{S R}-\Delta)$ and $(\overline{S R}+\Delta, \infty)$, its average re-projection error should be larger than the average re-projection error in $[\overline{S R}-\Delta, \overline{S R}+\Delta]$ that only contains the correct matches. Hence, the average re-projection errors in terms of the three intervals are calculated respectively, as shown in Fig. 3 and Table 1. Apparently, the matches in interval $[0.95,1.05]$ are more possible to be correct, since the average re-projection error for this interval is the smallest for any rotation angle mode. Notably, the average re-projection error for interval $[0.95,1.05]$ is slightly larger than zero due to feature locations errors, though its theoretical value should be equal to 0 .

Table 1. The average re-projection errors in different intervals for different rotation angles (in pixel)

\begin{tabular}{|c|c|c|c|c|c|c|}
\hline $\boldsymbol{S R}$ & $\mathbf{1 5}^{\circ}$ & $\mathbf{4 5}^{\circ}$ & $\mathbf{9 0}^{\circ}$ & $\mathbf{1 3 5}^{\circ}$ & $\mathbf{1 8 0}^{\circ}$ & $\mathbf{3 1 5}^{\circ}$ \\
\hline $\mathbf{( 0 , 0 . 9 5 )}$ & 9.605418297 & 8.612312417 & 40.76701386 & 4.076795974 & 3.625050846 & 10.50535836 \\
\hline$[\mathbf{0 . 9 5 , 1 . 0 5}]$ & $\mathbf{1 . 6 7 1 2 2 4 4 7 3}$ & $\mathbf{1 . 4 4 2 7 2 2 4 4 8}$ & $\mathbf{1 . 2 3 6 1 0 5 0 5 8}$ & $\mathbf{1 . 4 7 5 6 1 4 8 3 8}$ & $\mathbf{1 . 0 1 7 9 7 9 9 5 2}$ & $\mathbf{1 . 7 3 2 0 2 5 1 3 1}$ \\
\hline$(\mathbf{1 . 0 5}, \infty)$ & 3.168073732 & 21.03549685 & 3.917112333 & 19.75391581 & 1.450856696 & 13.59347741 \\
\hline
\end{tabular}

Observation B (Zoom): The target images are generated by resampling the reference image along width and height with the same scale variation $(S V)$. In particular, the $S V$ is set to be 0.5 (the target image is of size $1600 \times 1280$ in pixel), 0.67 (the target image is of size $1200 \times 960$ in pixel), 1.067 (the 
target image is of size $750 \times 600$ in pixel), 1.333 (the target image is of size $600 \times 480$ in pixel), 2.0 (the target image is of size $400 \times 320$ in pixel), and 4.0 (the target image is of size $200 \times 160$ in pixel), respectively. Six $S R$ distributions with different $S V s$ are shown in Fig. 4. According to our earlier argument, the $S R$ of a correct match must be equal to its corresponding $S V$. Similarly, for each zoom mode, all the $S R$ are divided into three intervals, i.e., $(0, S V-\Delta),[S V-\Delta, S V+\Delta]$, and $(S V+\Delta, \infty)$. For all the six modes, almost all the matches in interval $[S V-\Delta, S V+\Delta](\Delta=0.05)$ are identified to be correct subjectively. Therefore, the $S R$ of a correct match is approximately equal to its corresponding $S V$.

Table 2. The average re-projection errors in different intervals for different zoom modes

\begin{tabular}{|c|c|c|c|c|c|c|}
\hline $\boldsymbol{S R}$ & $\mathbf{0 . 5}$ & $\mathbf{0 . 6 7}$ & $\mathbf{1 . 0 6 7}$ & $\mathbf{1 . 3 3 3}$ & $\mathbf{2 . 0}$ & $\mathbf{4 . 0}$ \\
\hline$(\mathbf{0}, S V-\mathbf{0 . 0 5})$ & 1.074701304 & 1.324763419 & 2.231343973 & 19.28596508 & 75.99481258 & 7.550215636 \\
\hline$[S V \mathbf{- 0 . 0 5}, S V+\mathbf{0 . 0 5}]$ & $\mathbf{0 . 4 0 0 5 8 2 7 1 6}$ & $\mathbf{0 . 7 9 3 2 2 8 8 7 7}$ & $\mathbf{0 . 3 6 7 4 0 4 6 7 9}$ & $\mathbf{0 . 7 1 2 1 7 1 3 3}$ & $\mathbf{0 . 7 3 5 9 3 9 1 2}$ & $\mathbf{2 . 1 3 7 6 5 2 6 7 9}$ \\
\hline$(S V+\mathbf{0 . 0 5}, \infty)$ & 84.88320764 & 18.52771762 & 18.65179004 & 5.711980171 & 1.02038484 & 2.662736304 \\
\hline
\end{tabular}

In addition, the average re-projection errors of three intervals are shown in Fig. 5 and Table 2. It turns out that the average re-projection error for interval $[S V-0.05, S V+0.05]$ is smaller than the average re-projection error for the other two intervals. Therefore, it can be concluded that the matches in $[S V-0.05, S V+0.05]$ are more possible to be correct.

Observation C (Complex transformation): In this observation, matching is carried out on two images with different viewpoints for the same painting as shown in Fig. 1 (a) and (b), respectively. In the overlapped parts of the images, the size of the same object is different. The $S R$ distribution of all the matches is shown in Fig. 6 and the value of $\overline{S R}$ is 1.27. All the matches in interval $[\overline{S R}-\Delta, \overline{S R}+\Delta]$ ( $\Delta=0.07$ ) have been subjectively identified to be correct (Fig. 7). The false matches outside of interval $[\overline{S R}-\Delta, \overline{S R}+\Delta]$ have been given in Fig. 8. Although the ground-truth transformation is 
unknown, the average re-projection error cannot be straightforwardly calculated, our subjective identification of correct matches also prefers the SVH method. This indicates, all the matches whose scale ratio is approximately equal to the same $\overline{S R}$ should be correct.

It is also noted that in observation $\mathrm{A}$ and $\mathrm{B}, \Delta$ is 0.05 , but in observation $\mathrm{C}, \Delta$ is 0.07 . The degree of deviation between the average scale ratio and the actual scale variation of the two images is quantized by using $\Delta$. In theory, $\Delta$ will become 0 if the average scale ratio and the actual scale variation of two images are exactly the same. In practice, however, $\Delta$ cannot be always equal to 0 . More specifically, in the above three key observations, the values of $\Delta$ are different, since the actual scale ratio ranges of the correct matches confirmed subjectively are different. For practical applications, determining the value of $\Delta$ remains to be an open problem.

The above observations illustrate that the $S R$ of correct matches is fundamentally consistent with the scale variation of the two matched images, regardless of rotation, zoom or complex transformation induced by parallax. Therefore, it is possible and desirable to divide all the matches into the potential correct and false matches in advance according to the SVH.

\section{B. Grouping based on SVH}

In the matching results, the correct and false matches are generally mixed together. According to the $\mathrm{SVH}$, the matches whose $S R$ lies in $[\overline{S R}-\Delta, \overline{S R}+\Delta]$ are highly possible to be in the potential correct group, and the rest of the matches will be in the potential false group. Apparently, different observation results will be obtained with different values of $\Delta$, but the optimal grouping can only be obtained with one proper value of $\Delta$.

In order to obtain the proper value of $\Delta, S R$ is assumed to follow a normal distribution by the Kolmogorov-Smirnov test [23], where the significance level of the normality test is set to be 0.05 . This statistical hypothesis test is implemented on a collection of 1000 image pairs (containing 2000 different images), which includes the images of the same scene with different viewpoint, the different zoomed images, the optical/infrared images, the aerial/ground images. In this collection, there are some benchmark images collecting from the well-known image datasets described in [24-28]. The transformation in these image pairs may be either affine or projective. The experimental results 
demonstrate that the $S R$ distribution of around $95.5 \%$ of the image pairs justify the hypothesis of normal distribution. Therefore, the random variable $S R$ is normally distributed with mean $\mu$ and variance $\sigma^{2}$, where $\mu$ is the average of the random variable $S R$. Due to the unavailability of the true mean value, $\mu$ can be empirically set to be equal to $\overline{S R}$.

Suppose there are $N$ matches $\left(U_{N}\right)$. According to the SVH, the potential correct group $U_{n}^{I}$ $\left(\left|U_{n}^{I}\right|=n, n<N\right)$ is simply the collection shown in the shaded region in Fig. 9. The shaded region represents the probability of the matches whose $S R s$ are less than $\Delta$ deviation away from $\mu$. The probability is equivalent to the area of the shaded region, which is denoted as $\Omega$.

$$
P\{\mu-\Delta \leq S R \leq \mu+\Delta\}=\Omega
$$

There is a positive hypothesis that the number of false matches is less than that of the correct matches. So at least half of the whole matches are considered to be correct. Hence, suppose $S R^{\prime}=\frac{S R-\overline{S R}}{\sigma}$ and $\Omega=50 \%$, it can be obtained

$$
P\left\{S R^{\prime} \leq \frac{\Delta}{\sigma}\right\}=(\Omega+1) / 2=75 \%
$$

The quantile of $75 \%$ percentile of the standard normal distribution is 0.67 . Then according to Eq. (3),

$$
\Delta=0.67 * \sigma
$$

Additionally, $\mu$ is different from $\overline{S R}$ in rigid sense. Thus $\Delta$ should be revised to accommodate this issue and the revised $\Delta$ is denoted as $\Delta_{\text {revised }}$.

$$
\Delta_{\text {revised }}=\min (\Delta, \varepsilon)
$$

where $\varepsilon$ is the revised variable. According to the different degrees of parallax in the two overlapped images, $\varepsilon$ is set to be different values. For small parallax, the shape and size of the same object in the two overlapped images are similar. As a result, the fluctuation range of $S R$ is often small and most matches will be correct. In this case, a proper value of $\varepsilon$ is good enough, where $\varepsilon$ is set to be 0.12 in our experiments. In contrast, for large parallax, the shape of the same object is quite different. The value of $\varepsilon$ is set to be $\alpha^{*} 0.5$, where $\alpha=\min \left(\overline{S R}-S R_{\min }, S R_{\max }-\overline{S R}\right)$, and $S R_{\min }$ and $S R_{\max }$ 
represent the minimum and maximum of $S R$, respectively. Moreover, it is assumed that half of the matches will be correct during the computation of $\Delta$, however, this assumption does not always hold. Since the number of correct matches cannot be precisely known in advance, and a $50 \%$ ratio will be generally assumed. Although the assumption cannot be always consistent with the practical situation, this issue would only have slight influent on $\Delta$. Remarkably, the grouping based on this assumption is rather helpful in removing parts of false matches, which as a result, will decrease the contamination levels and increase the possibility of obtaining optimal model.

Finally, the correct matches with $S R$ distributing in $\left[\overline{S R}-\Delta_{\text {revised }}, \overline{S R}+\Delta_{\text {revised }}\right]$ will be grouped into $U_{n}^{I}$ and the false matches will be grouped into $U_{N-n}^{O}=U_{N}-U_{n}^{I}\left(\left|U_{N-n}^{O}\right|=N-n\right)$. Due to the deviation of $\Delta_{\text {revised }}$, there inevitably exist a few correct matches in $U_{N-n}^{O}$ and a few false matches in $U_{n}^{I}$, respectively. It is guaranteed that the probability of one match from $U_{n}^{I}$ being correct is higher than the one from $U_{N-n}^{O}$. Moreover, sorted $U_{n}^{I}$ and $U_{N-n}^{O}$ are denoted as $U_{(n)}^{I}$ and $U_{(N-n)}^{O}$, where the sorting is carried out in ascending order by the value of $\left|S R_{i}-\overline{S R}\right|$ with $S R_{i}$ representing the $S R$ of the $i$ th match. In each sorted group, higher ranking of the match would indicate higher probability for the match to be correct. In fact, the sorted groups will play a critical role in the homography estimation procedure, which will be described in detail in the next section.

\section{SVH_RANSAC}

In this section, the SVH-RANSAC, an improved version of RANSAC by potential correct and false matches grouping, is proposed to achieve additional robustness in high levels of contamination and with fast computational speed. Since the proposed method explores the scale restriction, it is more adaptable to planar homography problem. For many other multi-view geometry problems, such as finding essential matrices (or fundamental matrices) or solving perspective-n-point problems (PnP problems), however, it will be slightly less adaptable. The framework of the proposed algorithm is given in Fig. 10. The novelty of the proposed SVH-RANSAC algorithm can be summarized in three main aspects. Initially, in hypothesis generation, the sampling is preferably restricted in $U_{n}^{I}$ rather than 
the whole set $U_{N}$ in order to reduce the contamination in the selected samples. Subsequently, each model is scored by the number of the partial identified false matches instead of all the correct matches. Due to this particular strategy, the computational complexity of the evaluation procedure could be greatly reduced, while reliable evaluation can still be maintained. Finally, the test set of termination criterion can be adaptively selected according to the contamination levels, where fast convergence can be achieved. In the following sections, we will describe the above-mentioned procedures in detail.

\section{A. Preferential Hypothesis Generation}

As a matter of fact, the uncontaminated samples should be selected as early as possible regardless of the contamination levels. It is because this strategy could not only contribute to obtaining the optimal parameters of the model, but also speeding up convergence of the algorithm.

Assume a sample contains at least $m$ matches. Then for $N$ matches, there exist $Q=\left(\begin{array}{l}N \\ m\end{array}\right)$ samples. Since the matches coming from $U_{n}^{I}$ are more likely to be correct, the samples entirely from $U_{n}^{I}$ should be selected with priority. However, there are still a few correct matches in $U_{N-n}^{O}$. So when there are no more satisfactory samples from $U_{n}^{I}$, the samples will be selected from $U_{N-n}^{o}$. Accordingly, the $Q$ samples can be further divided into three parts $\Theta_{(j)}(j=1,2,3)$. $\Theta_{(1)}$ only consists samples entirely from $U_{n}^{I}$ and $\Theta_{(3)}$ only consists of the samples entirely from $U_{N-n}^{O}$. The rest of the samples are in $\Theta_{(2)}$. The sampling priority is set to be $\Theta_{(1)}>\Theta_{(2)}>\Theta_{(3)}$, where the samples from the same $\Theta_{(j)}$ are selected randomly. In general, the optimal model can be calculated by the samples from $\Theta_{(1)}$ and the sampling will extend to $\Theta_{(2)}$ and $\Theta_{(3)}$ only in the worst scenario.

\section{B. Fast and Reliable Hypothesis Evaluation}

In the existing literature, there is still no better trade-off between the evaluation reliability and speed.

For the existing evaluation methods, each model must be evaluated for all the matches in order to obtain reliable evaluation results. Even if the current model has been proved to be the optimal, the evaluation process cannot be terminated halfway. On the contrary, during evaluation, if the given model has been already worse than the current optimal model, there is no need to go through the rest of 
the evaluation any longer. Motivated by these observations, we propose a new hypothesis evaluation method that is fast and reliable.

There are three key ingredients in the proposed method. Firstly, the number of false matches corresponding to the optimal model must be recorded as the current minimum. During the evaluation process for any given model, the number of false matches instead of correct matches will be sequentially observed and will be compared with the current minimum record. As long as it surpasses the current minimum record, the model is rejected directly, and the rest of the evaluation work will be automatically cancelled. In contrast, if a model is not rejected halfway, it will be the optimal model so far. Secondly, since the lower-rank of a match in $U_{(n)}^{I}$ and $U_{(N-n)}^{o}$ is, the higher the probability of being false is. It will be helpful to determine a false match for premature termination of the evaluation. Therefore, each model will be evaluated starting from the back of $U_{(n)}^{I}$ and $U_{(N-n)}^{o}$. Thirdly, any given model is evaluated on $U_{(n)}^{I}$ and $U_{(N-n)}^{O}$, respectively, and the two evaluation results are recorded as guidance in testing the termination criterion.

Based on the above improvements, the proposed method can always return reliable evaluation results, which is equivalent to the model evaluation using all the matches. Only if a given model is temporarily accepted, all the matches will take part in the evaluation process. For other cases, the unnecessary evaluation can be reduced. In this way, both reliability and fast speed can be obtained.

\section{Termination Criterion based on the Contamination Level}

Notably, the homography estimation is an iterative process. In each iteration, termination criterion is tested to keep the balance between the convergence rate and possibility of obtaining a better model. Although improvements on hypothesis generation have been introduced in previous section, it is desirable that the optimal model is obtained as early as possible. This would become a very challenging task when the contamination levels are high since it would introduce a number of unnecessary 
iterations. In order to reduce these unnecessary iterations, a different test of termination criterion is proposed.

Convergence rate is regulated by $k_{\max }$ according to maximality in Eq. (6) [7]. Maximality could guarantee the probability of obtaining a better model after $k_{\max }$ samples is smaller than $\eta_{0}$ (set to $1 \%$ ).

$$
k_{\max }=\log \left(\eta_{0}\right) / \log \left(1-I_{N} / \partial\right)
$$

where $\partial$ is the cardinal of the test set and $I_{N}$ is the number of correct matches in the test set. For the original maximality, $U_{N}$ is used as the test set [7]. If the contamination level in $U_{N}$ is high, slow convergence will occur due to expansion of $k_{\max }$. Undoubtedly, a number of iterations will be unnecessary. According to (6), $k_{\max }$ is proportional to $1-I_{N} / \partial$, which is the contamination level of the test set. Therefore, in high contamination levels of $U_{N}$, it is intuitive to select a proper test set with low level of contamination in order to reduce $k_{\max }$.

According to Bayes theorem, the contamination level of $U_{N}$ can be estimated by the conditional probability $P\left(U_{N-n}^{O} \mid o\right)$ of a false match $o$ coming from $U_{N-n}^{O}$, which can be defined as

$$
P\left(U_{N-n}^{o} \mid o\right)=\frac{P\left(o \mid U_{N-n}^{o}\right)(N-n) / N}{P\left(o \mid U_{N-n}^{O}\right)(N-n) / N+P\left(o \mid U_{n}^{I}\right) n / N}
$$

where $P\left(U_{N-n}^{O} \mid o\right)$ and $P\left(o \mid U_{n}^{I}\right)$ reprensent the contamination level of $U_{N-n}^{O}$ and $U_{n}^{I}$, respectively. When $P\left(U_{N-n}^{O} \mid o\right)$ is larger than the threshold (set to be 0.5 based on the previous positive hypothesis), it indicates most matches in $U_{N-n}^{O}$ are false and the contamination level of $U_{N}$ is high. Generally, the contamination level of $U_{n}^{I}$ is lower than $U_{N}$. In this case, therefore, the test set will be substituted by $U_{n}^{I}$ and the number of iterations can be reduced.

Overall, the test set is $U_{n}^{I}$ when the contamination level of $U_{N}$ is high. Otherwise, the test set is still $U_{N}$. And for all the acquired models, the one that results in the termination for the first time will be chosen. 
Additionally, non-randomness [7] is used to prevent the cases where an incorrect model is selected.

According to non-randomness, the minimal number of required correct matches $I_{\min }$ can be calculated

by,

$$
I_{\text {min }}=\min \left\{j: \sum_{i=j}^{\partial} \beta^{i-m}(1-\beta)^{\partial-i+m}\left(\begin{array}{l}
\partial-m \\
i-m
\end{array}\right)<\Psi\right\}
$$

In (8), $\beta$ is the probability that an incorrect model calculated from a random sample contains a false match is supported by a match not included in this sample [7]. $\Psi$ is a probability threshold, where the probability of actual correct matches are accidentally identified to be correct by an arbitrary incorrect model is smaller than $\Psi$ [7]. $I_{\min }$ is proportional to the cardinal of the test set. Following the above-described test strategy, when the contamination level of $U_{N}$ is high, $U_{n}^{I}$ will be used as the test set. Subsequently, $I_{\min }$ will be reduced. Thereafter, the probability of rejecting a correct model will be reduced.

\section{Experiments and Discussion}

In this section, the proposed algorithm has been tested on a dataset consisting of 158 pairs of images. In this dataset, 75 pairs are collected from the AdelaideRMF dataset [24], the ISPRS Dataset [25], the Oxford University Dataset [26], the Zurich Dataset [27], and other challenging pairs [28]. The rest of the dataset consists of actual ground monitoring images and aerial images. It includes indoor and outdoor scenes, where different imaging conditions, such as viewpoint, scale, and illumination are covered. In the scenario of smoothly moving or fixed position of a camera, the overlapped images in all the cases can be related by a planar homography. The proposed algorithm is compared with three representative algorithms, i.e., RANSAC [2], PROSAC [7], and LO+-RANSAC [18]. Among these representative algorithms, RANSAC is the ancestor, where its descendants should perform better than it in principle. The RANSAC code is provided by Rob Hess ${ }^{2}$. PROSAC performs well in selecting the uncontaminated samples based on the similarity of textures. The PROSAC code is provided by ZG

\footnotetext{
${ }^{2}$ http://robwhess.github.io/opensift/
} 
$\mathrm{Tan}^{3}$. The $\mathrm{LO}^{+}-\mathrm{RANSAC}$, is provided by Karel Lebeda [18], featured at rapid processing. The parameters are set according to the guidelines in [29]. The existing RANSAC with scale restriction assumes the potential correct features are similar in scale. And its performance is limited in the situation of parallax. For fairness, it is not chosen to be compared with the proposed algorithm. For the four algorithms, the probability of the final optimal model corrupted by false matches is 0.01 , and the location error threshold for distinguishing correct matches and false matches is 1.0. Since the randomized algorithm returns different outputs every time, the final result has been averaged over 10,000 runs for fairness and accuracy. All the algorithms have been run on Intel Core i3-3110M 2.4

GHz four core computer with 4 GB RAM.

In this section, performance of the four algorithms has been compared from three main perspectives, i.e., robustness in contamination, computational speed, and location error of correct matches.

\section{A. Robustness to the Different Contamination Levels}

In practice, the contamination level varies with the different image pairs. When the samples are highly contaminated, the estimation should be exhausted on most samples in order to obtain a correct model, yielding an increased number of unnecessary iterations, which is not desired. Therefore, the performance of quickly returning the correct matches will be tested. In this case, the robustness is measured by the average number of correct matches $(\bar{I})$ and the average number of iterations $(\bar{S})$.

Generally, higher robustness will be achieved with larger $\bar{I}$ and smaller $\bar{S}$. In this section, the comparison of the robustness is carried out on both synthetic data and the real data, respectively.

First, five individual tests have been carried out on different synthetic data with different levels of contamination (data A: $50 \%$, data B: $60 \%$, data C: $70 \%$, data D: $80 \%$, and data E: $90 \%$ ). The cardinal of each synthetic data is 100 . In Table 3 , it is demonstrated that the four algorithms almost have the

\footnotetext{
${ }^{3}$ https://code.google.com/
} 
same $\bar{I}$. However, with an increase in contamination levels from data A to data $\mathrm{E}$, the other three algorithms would cost more iterations than the proposed algorithm. Therefore, the robustness of the proposed algorithm performs the best in this experiment.

Table 3. Comparison of the robustness in different contamination levels

\begin{tabular}{|c|c|c|c|c|c|}
\hline \multicolumn{2}{|c|}{ Data } & RANSAC & PROSAC & LO ${ }^{+}$RANSAC & Prop. \\
\hline \multirow{3}{*}{ A:50\% } & $\bar{S}$ & 177.152 & 121.34 & 65.37 & $\mathbf{2 4 . 3 6}$ \\
\cline { 2 - 6 } & $\bar{I}$ & 46.09 & 44.61 & 48.93 & 46.57 \\
\hline \multirow{3}{*}{ B:60\% } & $\bar{S}$ & 502.72 & 277.52 & 178.17 & $\mathbf{3 0 . 2 9}$ \\
\cline { 2 - 6 } & $\bar{I}$ & 34.94 & 35.85 & 37.98 & 35.42 \\
\hline \multirow{2}{*}{ C:70\% } & $\bar{S}$ & 1208.04 & 775.01 & 515.59 & $\mathbf{3 5 . 5 6}$ \\
\cline { 2 - 6 } & $\bar{I}$ & 28.68 & 28.02 & 28.9 & 28.82 \\
\hline \multirow{3}{*}{ D:80\% } & $\bar{S}$ & 8779.51 & 3531.85 & 2396.55 & $\mathbf{1 2 8 . 4 8}$ \\
\cline { 2 - 6 } & $\bar{I}$ & 16.79 & 19.00 & 19.51 & 19.36 \\
\hline \multirow{2}{*}{ E:90\% } & $\bar{S}$ & 90310.46 & 49159.28 & 49875.02 & $\mathbf{2 4 7 . 9 1}$ \\
\cline { 2 - 6 } & $\bar{I}$ & 9.10 & 9.87 & 8.82 & 9.92 \\
\hline
\end{tabular}

Besides, in real-time applications, the number of iterations is commonly restricted. In this case, the four algorithms provide different performance. The experiments have been tested on data D and data E, where more iterations are required for high levels of contaminated data. The maximum number of iterations is set to be 500, 1000, 1500, 2000, and infinity. The results are given in Fig. 11. Thereinto, minI, maxI, and AvgI denote the obtainable minimum, maximum, and average number of the returned correct matches in the prescriptive iterations, respectively. The number of the returned correct matches in each iteration fluctuates in the range $[\min I, \max I]$. With equal number of iterations, the algorithm returning the largest $A v g I$ is the most robust one. For RANSAC and $\mathrm{LO}^{+}-\mathrm{RANSAC}, A v g I$ is proportional to the number of iterations, and the outputs of the two algorithms are not stable when the contamination levels become high. In contrast, AvgI returned by PROSAC is more stable and is close to maxI . Comparatively, the proposed algorithm returns almost the same number of correct matches as the other algorithms, but with much less number of iterations as shown in Table 3. For Data 
$\mathrm{D}$, the average number of iterations for the proposed algorithm is 128.48 . For Data $\mathrm{E}$, the average number of iterations for the proposed algorithm is 247.91. For these two dataset, the number of iterations is clearly less than 500. It should be noted that all the algorithms could return the maximum value of the correct matches since they are all random methods. Therefore, it is possible that the returned maxI by any one of the four algorithms with different prescriptive iterations could still be the same.

Table 4. Comparison of matching results in the case of small parallax

\begin{tabular}{|c|c|c|c|c|c|c|c|}
\hline \multicolumn{2}{|c|}{ Image Pair } & \multirow{2}{*}{$\begin{array}{c}\boldsymbol{R A N S A C} \\
325.76 \\
(252 \sim 367)\end{array}$} & \multirow{2}{*}{$\begin{array}{c}\text { PROSAC } \\
275.78 \\
(215 \sim 343) \\
\end{array}$} & \multirow{2}{*}{\begin{tabular}{|c|}
$\boldsymbol{O}^{+}-\boldsymbol{R A N S A C}$ \\
$\mathbf{4 0 6}$ \\
$(406 \sim 406)$
\end{tabular}} & \multirow{2}{*}{\begin{tabular}{|c|} 
Prop. \\
359.55 \\
$(319 \sim 369)$ \\
\end{tabular}} & \multirow{4}{*}{$\begin{array}{c}\begin{array}{c}\text { Saved } \\
\text { Iteration } \\
(\%)\end{array} \\
\\
45.03\end{array}$} & \multirow{4}{*}{$\begin{array}{c}\begin{array}{c}\text { Saved } \\
\text { Evaluation } \\
(\%)\end{array} \\
\\
90.05\end{array}$} \\
\hline \multirow{3}{*}{$\begin{array}{c}\text { Boston } \\
1712 \times 1368 \\
\mathrm{MN}=493\end{array}$} & $\bar{I}$ & & & & & & \\
\hline & $\bar{S}$ & $\begin{array}{c}47.34 \\
(15 \sim 133) \\
\end{array}$ & $\begin{array}{c}52.35 \\
(24 \sim 132) \\
\end{array}$ & \begin{tabular}{|c|}
23.36 \\
$(8 \sim 53)$ \\
\end{tabular} & $\begin{array}{c}\mathbf{1 2 . 8 4} \\
(6 \sim 40) \\
\end{array}$ & & \\
\hline & $\bar{S} \times T_{V}$ & 21788.33 & 11350.78 & 39008.53 & 3881.12 & & \\
\hline \multirow{3}{*}{$\begin{array}{c}\text { City } \\
329 \times 278 \\
\mathrm{MN}=28\end{array}$} & $\bar{I}$ & $\begin{array}{c}17.97 \\
(16 \sim 20) \\
\end{array}$ & $\begin{array}{c}16.37 \\
(11 \sim 18)\end{array}$ & $\begin{array}{c}25 \\
(25 \sim 25)\end{array}$ & $\begin{array}{c}18.82 \\
(15 \sim 21)\end{array}$ & \multirow{3}{*}{61.69} & \multirow{3}{*}{92.96} \\
\hline & $\bar{S}$ & $\begin{array}{c}34.69 \\
(16 \sim 54)\end{array}$ & $\begin{array}{c}46.37 \\
(32 \sim 192)\end{array}$ & $\begin{array}{c}17.67 \\
(4 \sim 51)\end{array}$ & $\begin{array}{c}\mathbf{6 . 7 7} \\
(3 \sim 17)\end{array}$ & & \\
\hline & $\bar{S} \times T_{V}$ & 947.15 & 653.24 & 2096.55 & 147.54 & & \\
\hline \multirow{3}{*}{$\begin{array}{c}\text { Ladysymon } \\
2272 \times 1704 \\
\mathrm{MN}=570\end{array}$} & $\bar{I}$ & $\begin{array}{c}126.29 \\
(96 \sim 142)\end{array}$ & $\begin{array}{c}122.72 \\
(86 \sim 141)\end{array}$ & $\begin{array}{c}\mathbf{1 7 2 . 2 5} \\
(172 \sim 175) \\
\end{array}$ & $\begin{array}{c}133.52 \\
(97 \sim 142)\end{array}$ & \multirow{3}{*}{40.25} & \multirow{3}{*}{57.39} \\
\hline & $\bar{S}$ & $\begin{array}{c}3346.58 \\
(1300 \sim 10241)\end{array}$ & $\begin{array}{c}2229.99 \\
(1228 \sim 8885)\end{array}$ & $\begin{array}{c}549.63 \\
(517 \sim 565) \\
\end{array}$ & $\begin{array}{c}\mathbf{3 2 8 . 4 3} \\
(170 \sim 587) \\
\end{array}$ & & \\
\hline & $\bar{S} \times T_{V}$ & 1818142.90 & 632233.22 & 294560.67 & 125522.01 & & \\
\hline \multirow{3}{*}{$\begin{array}{c}\text { Build } \\
640 \times 480 \\
M N=452\end{array}$} & $\bar{I}$ & $\begin{array}{c}352.04 \\
(322 \sim 364)\end{array}$ & $\begin{array}{c}297.92 \\
(213 \sim 364)\end{array}$ & $\begin{array}{c}\mathbf{4 0 0} \\
(400 \sim 400) \\
\end{array}$ & $\begin{array}{c}361.02 \\
(354 \sim 364)\end{array}$ & \multirow{3}{*}{49.83} & \multirow{3}{*}{94.19} \\
\hline & $\bar{S}$ & $\begin{array}{c}18.78 \\
(10 \sim 35) \\
\end{array}$ & $\begin{array}{c}32.99 \\
(13 \sim 105) \\
\end{array}$ & $\begin{array}{c}11.84 \\
(5 \sim 41) \\
\end{array}$ & \begin{tabular}{|c|}
$\mathbf{5 . 9 4}$ \\
$(3 \sim 15)$ \\
\end{tabular} & & \\
\hline & $\bar{S} \times T_{V}$ & 9213.70 & 6658.33 & 32648.23 & 1895.40 & & \\
\hline \multirow{3}{*}{$\begin{array}{c}\text { Castle } \\
768 \times 576 \\
\mathrm{MN}=43\end{array}$} & $\bar{I}$ & $\begin{array}{c}16.71 \\
(12 \sim 19) \\
\end{array}$ & $\begin{array}{c}14.92 \\
(12 \sim 18) \\
\end{array}$ & $\begin{array}{c}\mathbf{2 0 . 9 6} \\
(18 \sim 23) \\
\end{array}$ & $\begin{array}{c}17.69 \\
(12 \sim 20) \\
\end{array}$ & \multirow{3}{*}{62.42} & \multirow{3}{*}{85.29} \\
\hline & $\bar{S}$ & $\begin{array}{c}311.17 \\
(148 \sim 757) \\
\end{array}$ & $\begin{array}{c}384.09 \\
(186 \sim 914) \\
\end{array}$ & $\begin{array}{c}76.82 \\
(52 \sim 145) \\
\end{array}$ & $\begin{array}{c}\mathbf{2 8 . 8 7} \\
(16 \sim 72) \\
\end{array}$ & & \\
\hline & $\bar{S} \times T_{V}$ & 13195.49 & 9006.83 & 5195.60 & 764.05 & & \\
\hline \multirow{3}{*}{$\begin{array}{c}\text { Corr } \\
512 \times 512 \\
\mathrm{MN}=177\end{array}$} & $\bar{I}$ & $\begin{array}{c}67.21 \\
(58 \sim 74) \\
\end{array}$ & $\begin{array}{c}64.25 \\
(56 \sim 73) \\
\end{array}$ & $\begin{array}{c}\mathbf{9 2 . 4 7} \\
(81 \sim 95) \\
\end{array}$ & $\begin{array}{c}70.21 \\
(50 \sim 78) \\
\end{array}$ & \multirow{3}{*}{39.13} & \multirow{3}{*}{80.08} \\
\hline & $\bar{S}$ & $\begin{array}{c}283.43 \\
(198 \sim 482) \\
\end{array}$ & $\begin{array}{c}274.98 \\
(157 \sim 471) \\
\end{array}$ & $\begin{array}{c}59.52 \\
(53 \sim 129) \\
\end{array}$ & $\begin{array}{c}\mathbf{3 6 . 2 3} \\
(23 \sim 61) \\
\end{array}$ & & \\
\hline & $\bar{S} \times T_{V}$ & 50531.84 & 24537.10 & 21776.38 & 4337.28 & & \\
\hline
\end{tabular}


Finally, the performance of the algorithm is tested on real data, where two classes are included. In the first class, the parallax in the two overlapped images is small, while the parallax is large in the second class. All the 158 pairs of images are involved in this comparison, and only representatives are given in Fig. 12 and Fig. 13 for brevity. These selected images are quite challenging for homography estimation, which have been used for evaluating their methods in other papers [7], [18]. Apart from parallax, the intensity of the same object in Fig. 12 (b), Fig. 13 (c), Fig. 13 (h), and Fig. 13 (j) are all different. The results in Table 4 and Table 5 indicate that the proposed algorithm can perform well even with $90 \%$ contamination level and can return the correct matches more quickly if compared with the other three algorithms.

Table 5. Comparison of matching results in the case of large parallax

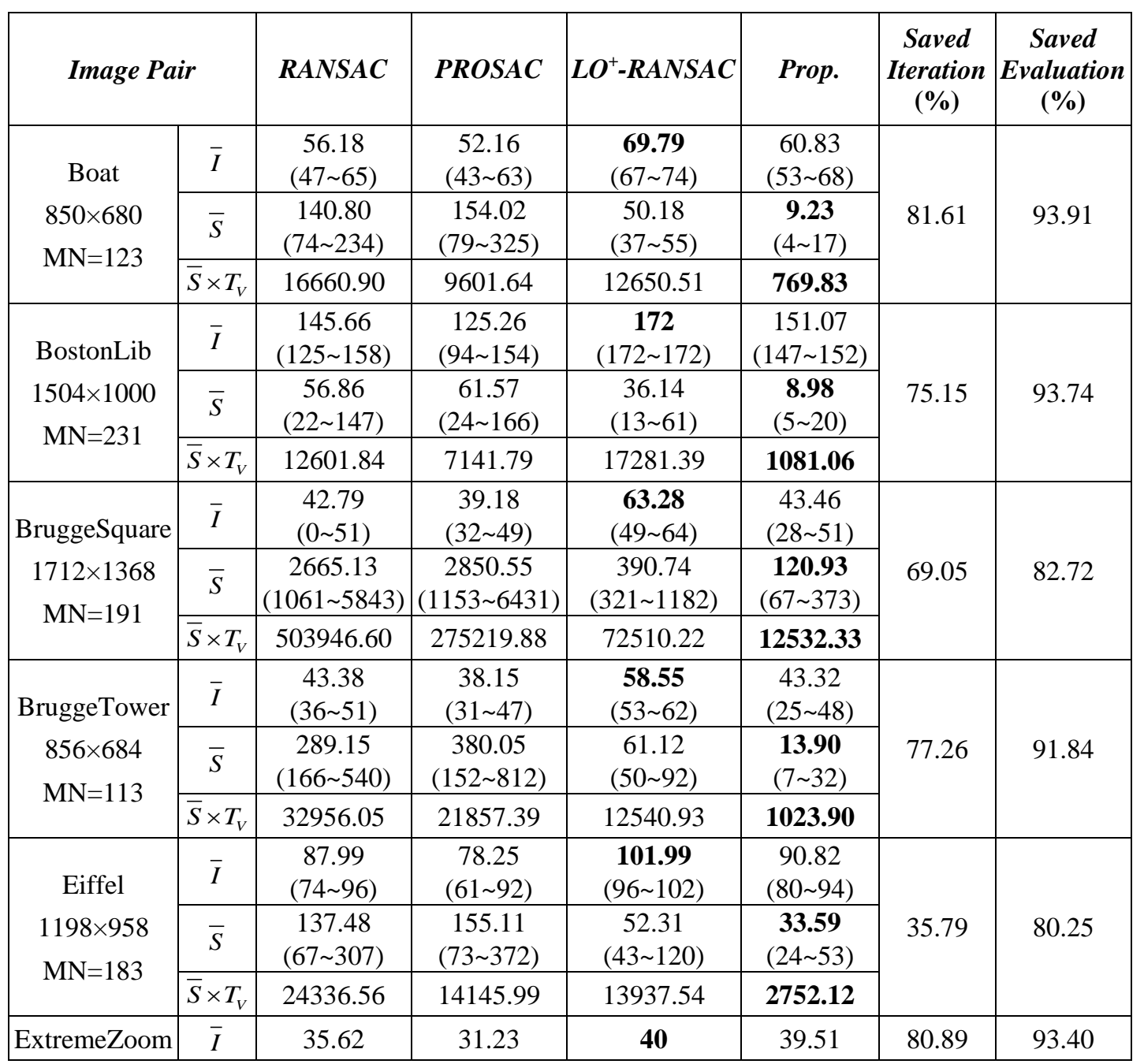




\begin{tabular}{|c|c|c|c|c|c|c|c|}
\hline \multirow{3}{*}{$\begin{array}{c}1519 \times 1006 \\
\mathrm{MN}=67\end{array}$} & & $(27 \sim 40)$ & $(24 \sim 39)$ & $(38 \sim 41)$ & $(37 \sim 40)$ & & \\
\hline & $\bar{S}$ & $\begin{array}{c}101.90 \\
(34 \sim 236)\end{array}$ & $\begin{array}{c}115.72 \\
(47 \sim 278)\end{array}$ & $\begin{array}{c}52.17 \\
(27 \sim 110)\end{array}$ & $\begin{array}{c}\mathbf{9 . 9 7} \\
(5 \sim 20)\end{array}$ & & \\
\hline & $\bar{S} \times T_{V}$ & 7131.67 & 3897.57 & 5065.74 & 334.20 & & \\
\hline \multirow{3}{*}{$\begin{array}{c}\text { Graffiti } \\
800 \times 640 \\
M N=176\end{array}$} & $\bar{I}$ & $\begin{array}{c}63.97 \\
(49 \sim 73)\end{array}$ & $\begin{array}{c}52.44 \\
(39 \sim 71)\end{array}$ & $\begin{array}{c}\mathbf{8 8 . 5 1} \\
(62 \sim 91)\end{array}$ & $\begin{array}{c}65.32 \\
(39 \sim 75)\end{array}$ & \multirow{3}{*}{75.63} & \multirow{3}{*}{91.30} \\
\hline & $\bar{S}$ & $\begin{array}{c}433.92 \\
(172 \sim 985)\end{array}$ & $\begin{array}{c}701.49 \\
(217 \sim 1982)\end{array}$ & $\begin{array}{c}69.91 \\
(62 \sim 297)\end{array}$ & $\begin{array}{l}\mathbf{1 7 . 0 4} \\
(9 \sim 34)\end{array}$ & & \\
\hline & $\bar{S} \times T_{V}$ & 72027.31 & 61931.46 & 23486.64 & 2043.09 & & \\
\hline \multirow{3}{*}{$\begin{array}{c}\text { Brussels } \\
1712 \times 1368 \\
\mathrm{MN}=849\end{array}$} & $\bar{I}$ & $\begin{array}{c}306.41 \\
(251 \sim 347) \\
\end{array}$ & $\begin{array}{c}279.68 \\
(218 \sim 343) \\
\end{array}$ & $\begin{array}{c}\mathbf{4 4 4 . 7 2} \\
(351 \sim 455) \\
\end{array}$ & $\begin{array}{c}305.62 \\
(137 \sim 351) \\
\end{array}$ & \multirow{3}{*}{45.22} & \multirow{3}{*}{83.93} \\
\hline & $\bar{S}$ & $\begin{array}{c}464.43 \\
(173 \sim 1001) \\
\end{array}$ & $\begin{array}{c}438.72 \\
(204 \sim 1131) \\
\end{array}$ & $\begin{array}{c}59.77 \\
(54 \sim 325) \\
\end{array}$ & $\begin{array}{c}\mathbf{3 2 . 7 4} \\
(13 \sim 99)\end{array}$ & & \\
\hline & $\bar{S} \times T_{V}$ & 365779.08 & 186078.11 & 104247.52 & 16756.54 & & \\
\hline \multirow{3}{*}{$\begin{array}{c}\text { GreatWall } \\
2272 \times 1704 \\
\mathrm{MN}=100\end{array}$} & $\bar{I}$ & $\begin{array}{c}9.20 \\
(8 \sim 14)\end{array}$ & $\begin{array}{c}10.66 \\
(8 \sim 13)\end{array}$ & $\begin{array}{c}\mathbf{1 5 . 3 9} \\
(10 \sim 16)\end{array}$ & $\begin{array}{c}10.79 \\
(9 \sim 15)\end{array}$ & \multirow{3}{*}{73.36} & \multirow{3}{*}{36.60} \\
\hline & $\bar{S}$ & $\begin{array}{c}18061.67 \\
\left(10^{4} \sim 46050\right)\end{array}$ & $\begin{array}{c}10^{4} \\
\left(10^{4} \sim 10^{4}\right)\end{array}$ & $\begin{array}{c}9278.32 \\
(7586 \sim 36479) \\
\end{array}$ & $\begin{array}{c}\mathbf{2 4 7 2 . 1 2} \\
(632 \sim 7229) \\
\end{array}$ & & \\
\hline & $\bar{S} \times T_{V}$ & 1806241.55 & 516329.96 & 179653.49 & 113894.55 & & \\
\hline \multirow{3}{*}{$\begin{array}{c}\text { Angiogram } \\
1024 \times 1024 \\
\mathrm{MN}=20\end{array}$} & $\bar{I}$ & $\begin{array}{c}4.18 \\
(3 \sim 6)\end{array}$ & $\begin{array}{c}0 \\
(0 \sim 0)\end{array}$ & $\begin{array}{c}\mathbf{6} \\
(6 \sim 6)\end{array}$ & $\begin{array}{c}\mathbf{6} \\
(6 \sim 6)\end{array}$ & \multirow{3}{*}{75.85} & \multirow{3}{*}{2.50} \\
\hline & $\bar{S}$ & $\begin{array}{c}427.56 \\
(305 \sim 567) \\
\end{array}$ & $\begin{array}{c}566.36 \\
(566 \sim 567) \\
\end{array}$ & $\begin{array}{c}636.0 \\
(636 \sim 673) \\
\end{array}$ & $\begin{array}{c}\mathbf{1 5 3 . 5 9} \\
(100 \sim 223) \\
\end{array}$ & & \\
\hline & $\bar{S} \times T_{V}$ & 8226.15 & 5846.53 & 935.28 & 911.88 & & \\
\hline \multirow{3}{*}{$\begin{array}{c}\text { WhiteBoard } \\
1504 \times 1000 \\
\mathrm{MN}=37\end{array}$} & $\bar{I}$ & $\begin{array}{c}18.79 \\
(15 \sim 21)\end{array}$ & $\begin{array}{c}16.26 \\
(13 \sim 20)\end{array}$ & $\begin{array}{c}\mathbf{2 5 . 9 9} \\
(18 \sim 26)\end{array}$ & $\begin{array}{c}20.13 \\
(20 \sim 22)\end{array}$ & \multirow{3}{*}{80.25} & \multirow{3}{*}{93.36} \\
\hline & $\bar{S}$ & $\begin{array}{c}97.56 \\
(51 \sim 223) \\
\end{array}$ & $\begin{array}{c}159.79 \\
(80 \sim 301)\end{array}$ & $\begin{array}{c}32.36 \\
(15 \sim 77) \\
\end{array}$ & $\begin{array}{c}\mathbf{6 . 3 9} \\
(4 \sim 10) \\
\end{array}$ & & \\
\hline & $\bar{S} \times T_{V}$ & 3424.78 & 2994.73 & 2616.47 & 173.80 & & \\
\hline
\end{tabular}

In the implementation of the proposed algorithm, less iterations are needed compared with PROSAC,

which indicates that SVH outperforms the similarity of the texture in the aspect of selecting the uncontaminated samples early. Moreover, the scale difference is obvious for large parallax, and the premise that the scale of two matched features should be equal [10-14] is not satisfied. The results shown in Table 3 demonstrate that the constraint on the scale of a match for SVH is more relaxed than the scale constraint in [10-14]. Additionally, for the case of "Angiogram", according to non-randomness, PROSAC cannot return any correct matches. However, both $\mathrm{LO}^{+}-\mathrm{RANSAC}$ and the proposed algorithm can return 6 correct matches. Notably, in the proposed algorithm, the cardinal of the potential correct group is 13 and $I_{\min }$ is 6 . Therefore, the proposed algorithm will not reject this 
correct model, which has demonstrated that the proposed algorithm can substantially reduce the probability of rejecting a correct model compared to PROSAC.

According to the extensive comparison from the above three perspectives, the proposed algorithm is demonstrated to be more robust for high level of contamination.

\section{B. Computation Speed}

The total execution time $T$ of the other three algorithms can be given by

$$
T=\bar{S}\left(T_{M}+T_{V}\right)=\bar{S} \times T_{M}+\bar{S} \times T_{V}
$$

And the computational time for SVH-RANSAC can thus be given by,

$$
T=T_{G}+\bar{S}\left(T_{M}+T_{V}\right)=T_{G}+\bar{S} \times T_{M}+\bar{S} \times T_{V}
$$

where $T_{M}$ is the time to compute the model parameters. Among the four algorithms, $T_{M}$ is identical.

$T_{V}$ represents the time spent on the hypothesis evaluation and is replaced by the average number of matches participating in evaluating a given model. $T_{G}$ represents the time consumed in dividing the potential correct and false matches, which should be proportional to the number of the matches. In general, $T_{G}$ is often a very small value., If $N$ is 800 , for example, $T_{G}$ is only $1 \mathrm{~ms}$. Thus, the speed of the four algorithms should be compared in $\bar{S}$ and $\bar{S} \times T_{V}$, where $\bar{S} \times T_{V}$ represents the average number of matches participating in the hypothesis evaluation for an output. Therefore, the smaller the values of $\bar{S}$ and $\bar{S} \times T_{V}$ are, the faster the algorithm will be.

Two types of matching results have been given in Table 4 and Table 5. In these two tables, MN is the number of the matches based on KNN. The best results are shown in bold and two numerical values in brackets are the minimum and maximum values. In the evaluation of computational speed, the proposed algorithm is compared with $\mathrm{LO}^{+}$-RANSAC, which has fastest computational speed in all the algorithms used for comparison, where the Saved iterations in Eq. (11) and the Saved evaluation in Eq. (12) are used. 


$$
\begin{gathered}
\text { Saved iteration }=\frac{\bar{S}_{L O^{+} \_R A N S A C}-\bar{S}_{\text {Prop. }}}{\bar{S}_{L O^{+}{ }_{-} R A N S A C}} \\
\text { Saved evaluation }=\frac{\left(\bar{S} \times T_{V}\right)_{L O^{+}-R A N S A C}-\left(\bar{S} \times T_{V}\right)_{P r o p .}}{\left(\bar{S} \times T_{V}\right)_{L O^{+}-R A N S A C}}
\end{gathered}
$$

These results have shown that the proposed algorithm can save at least by $35 \%$ in the number of iterations. For each output, at least $2.5 \%$ evaluation computation can be saved. Even when the contamination level is more than $80 \%$, such as "GreatWall" in our experiments, the proposed algorithm can still save up to $73.36 \%$ in the number of iterations and $36.60 \%$ evaluation consumption. The results demonstrate that computational speed of proposed algorithm is faster than that of the $\mathrm{LO}^{+}$-RANSAC.

\section{Location error of the Returned Correct Matches}

In the existing homography estimation algorithms, the average number of the returned correct matches has been widely used as a metric for performance evaluation. However, apart from this quantitative metric, the location error of the returned correct matches is another important factor to be considered. In this sense, a highly precise model should be the one with correct matches as well as low location errors.

The four algorithms used in comparison can return a set of correct matches, and each correct match corresponds to a re-projection error as given in Fig. 14. The value of the re-projection error ranges from zero to infinity in pixel. Since the feature position is usually an integer in computer representations, the matches whose re-projection errors are smaller than half of pixel will not increase the feature location error. In contrast, the matches whose re-projection error is larger than one pixel will increase the feature location error, where the precision of the model will be compromised. The less the re-projection error is, the better the matcher will be. For this comparison, all the returned correct matches are divided into three intervals $(0,0.5),[0.5,1.0]$ and $(1.0, \infty)$ in pixel according to their re-projection errors. In general, the algorithm with more matches returned in $(0,0.5)$ and fewer matches returned in intervals 
$[0.5,1.0]$ and $(1.0, \infty)$, is considered to be better for the precision of the returned model. In order to obtain comprehensive evaluation results, TotalRE, indicating the total location error of all the returned correct matches, is defined in Eq. (13), where TotalRE is obtain by sum of errors in three different intervals. $P N_{(0,0.5)}, P N_{[0.5,1.0]}$ and $P N_{(1.0, \infty)}$ respectively represent the percentage of the returned correct matches whose re-projection error (pixel) locates in $(0,0.5),[0.5,1.0]$ and $(1.0, \infty)$. The average re-projection errors of the three intervals are represented by $A R E_{(0,0.5)}, A R E_{[0.5,1.0]}$ and $A R E_{(1.0, \infty)}$, respectively. In general, lower value of TotalRE implies better performance of the algorithm.

$$
\text { TotalRE }=P N_{(0,0.5)} \times A R E_{(0,0.5)}+P N_{[0.5,1.0]} \times A R E_{[0.5,1.0]}+P N_{(1.0, \infty)} \times A R E_{(1.0, \infty)}
$$

The matching of the "Graffiti" (Fig. 2 (a)) and its $45^{\circ}$ rotation image, for example, the corresponding average re-projection error ( $A R E$ ) for each interval is given in Table 6. In evaluation of TotalRE, the proposed algorithm could achieve 73.36, which is much less than other three algorithms. In general, the proposed algorithm could achieve lowest location error for the returned correct matches.

Table 6. The total location error of the returned correct matches in four algorithms for $45^{\circ}$ rotation

\begin{tabular}{|c|c|c|c|c|c|}
\hline \multicolumn{2}{|c|}{ location error $($ pixel) } & RANSAC & PROSAC & LO $\boldsymbol{H}^{+}$RANSAC & Prop. \\
\hline \multirow{2}{*}{$(0,0.5)$} & $P N_{(0,0.5)}$ & $10.43 \%(63)$ & $13.15 \%(78)$ & $13.34 \%(83)$ & $13.16 \%(\mathbf{8 0})$ \\
\cline { 2 - 6 } & $A R E_{(0,0.5)}$ & 0.33 & 0.35 & 0.35 & $\mathbf{0 . 3 5}$ \\
\hline \multirow{2}{*}[0.5,1.0]{} & $P N_{[0.5,1.0]}$ & $65.07 \%(393)$ & $77.07 \%(457)$ & $74.28 \%(462)$ & $76.15 \%(\mathbf{4 6 3})$ \\
\cline { 2 - 6 } & $A R E_{[0.5,1.0]}$ & 0.75 & 0.74 & 0.74 & $\mathbf{0 . 7 4}$ \\
\hline \multirow{2}{*}{$(1.0, \infty)$} & $P N_{(1.0, \infty)}$ & $24.50 \%(148)$ & $9.78 \%(58)$ & $12.38 \%(77)$ & $10.69 \%(65)$ \\
\cline { 2 - 6 } & $A R E_{(1.0, \infty)}$ & 6.89 & 2.65 & 1.24 & $\mathbf{1 . 1 6}$ \\
\hline \multirow{2}{*}{ The total number of returned } & 604 & 593 & 622 & $\mathbf{6 0 8}$ \\
\hline \multicolumn{2}{r|}{ correct matches } & 221.05 & 87.55 & 74.99 & $\mathbf{7 3 . 3 6}$ \\
\hline
\end{tabular}

\section{Limitation and discussion}


For RANSAC, the global optimal model of one plane can only be obtained with the largest number of correct matches. In other words, the proposed method, as a variant of RANSAC, is not applicable for many other multi-view geometry problems such as estimating essential matrices (or fundamental matrices) or solving perspective-n-point problems ( $\mathrm{PnP}$ problems), where its strength remains in finding planar homography. Even though the proposed algorithm are tested on some of images such as "Castle" and "Ladysymon" with 3D structures for validation purposes, the success of the proposed method largely depends on the fact that these images could be approximated to a single plane.

In summary, the proposed SVH-RANSAC could obtain improved robustness in high levels of contamination and achieve the fast computational speed. For image matching tasks, in particular, the proposed algorithm could obtain promising results as shown in our experiments.

\section{Conclusion}

This paper proposes the scale variation homogeneity, which is more robust than the similarity of feature texture and other scale restrictions for identify a potential correct match. Based on SVH, a new method, i.e., SVH-RANSAC, is proposed in this paper. As a variant of the original RANSAC, SVH-RANSAC restricts the sampling preferentially from the potential correct matches, and scores each model according to the numbers of false matches in order to quickly obtain reliable evaluation results, and selects the proper test set adaptively to calculate the termination criterion for a fast convergence. The experiments have demonstrated that the proposed scheme could achieve promising results, particularly in improving robustness for high levels of contamination and with rapid computational speed. In fact, the value of $\Delta$ is related to the degree of parallax. In our future work, the relationship between the degree of parallax and the scale changes in two matched images will be investigated to provide potential guidance for the setting of $\Delta$.

\section{APPENDIX A}

Computation of the re-projection error

The re-projection error of a correspondence is a geometric error representing the image distance between a projected point and a measured one. Suppose that $\left(x, x^{\prime}\right)$ represents one correspondence, 
$x$ is the referenced feature coordinate, $\boldsymbol{x}^{\prime}$ is the matched feature coordinate and $\hat{x}$ is the true projection coordinate of $\boldsymbol{x}^{\prime}$ by the homography $\boldsymbol{H}$. Due to the presence of errors, the homography is compromised, namely $\boldsymbol{x}^{\prime} \boldsymbol{H} \neq \boldsymbol{x}$ and $\boldsymbol{x}^{\prime} \boldsymbol{H}=\hat{\boldsymbol{x}}$. The re-projection error for $\left(x, \boldsymbol{x}^{\prime}\right)$ is simply the distance between $x$ and $\hat{x}$. Thus the re-projection error for one correspondence can be defined as

$$
R E=d(\boldsymbol{x}, \hat{\boldsymbol{x}})
$$

where $\hat{\boldsymbol{x}}$ is computed according to the actual rotational angle (or zoom) since $\boldsymbol{H}$ is unavailable and $\boldsymbol{d}$ is the Euclidean distance. Furthermore, average re-projection error for $N$ correspondences can be defined as

$$
A R E=\left(\sum_{i=1}^{N} R E_{i}\right) / N
$$

\section{ACKNOWLEDGMENT}

The authors appreciate Mr. K. Lebeda for providing the code of the $\mathrm{LO}^{+}-\mathrm{RANSAC}$. This work was partially supported by the National 863 Program (Project No. 2014AA015104), the National Science Foundation of China (No. 61370124) and the National Science Fund for Distinguished Young Scholars (No. 61125206).

\section{References}

[1] D. G. Lowe, "Distinctive image features from scale-invariant keypoints," Int. J. Comput. Vis., vol. 2, no. 60, pp. 91-110, 2004.

[2] M. A. Fischler and R. C. Bolles, "Random sample consensus: A paradigm for model fitting with applications to image analysis and automated cartography," Commun. ACM, vol. 24, pp. 381-395, Jun.1981.

[3] J. Matas and O. Chum, "Randomized RANSAC with Td,d test," Image Vis. Comput., vol. 22, no. 10, pp. 837-842,2004.

[4] O. Chum and J. Matas, "Optimal randomized RANSAC," IEEE Trans. Pattern Anal. Mach. Intell., vol. 30, no. 8, pp. 1472-1482, 2008.

[5] K. Lebeda, J. Matas, and O. Chum, "Fixing the Locally Optimized RANSAC," in Proc. Brit. Mach. Vis. Conf., 2012, pp. 95.1-95.11. 
[6] O. Chum, J. Matas, and J. Kittler, "Locally optimized RANSAC," in Proc. Ann. Symp. German Assoc. for Pattern Recognition, 2003, pp. 236-243.

[7] O. Chum and J. Matas, "Matching with PROSAC-progressive sample consensus," in Proc. IEEE Conf. Comput. Vis. Pattern Recognit., 2005, pp. 220-226.

[8] T. Botterill, S. Mills, and R. Green, "New Conditional Sampling Strategies for Speeded-Up RANSAC,” in Proc. Brit. Mach. Vis. Conf., 2009, pp. 33.1-33.11.

[9] A. Meler, M. Decrouez, and J. Crowley, "BetaSAC: a new conditional sampling for RANSAC," in Proc. Brit. Mach. Vis. Conf., 2010, pp. 42.1-42.11.

[10] Z. Yi, C. Zhiguo, and X. Yang, "Multi-spectral remote image registration based on SIFT," Electron. Lett., vol. 44, no. 2, pp. 107-108, Jan. 2008.

[11] Q. Li, G. Wang, J. Liu, and S. Chen, "Robust Scale-Invariant Feature Matching for Remote Sensing Image Registration,” IEEE Geosci. Remote Sens. Lett., vol. 6, no. 2, pp. 287-291, Apr. 2009.

[12] M.F. Vural, Y. Yardimci, and A. Temizel, "Registration of multispectral satellite images with Orientation-Restricted SIFT,” in Proc. IEEE Int. Geosci. Remote Sens. Symp., Jul. 2009, vol.3, pp. $243-246$.

[13] Y. Bastanlar, A. Temizel and Y. Yardimci, "Improved SIFT matching for image pairs with scale difference,” Electron. Lett., vol. 46, no. 5, pp. 346-348, Mar. 2010.

[14] Q. Xu, Y. Zhang and B. Li, "Improved SIFT match for optical satellite images registration by size classification of blob-like structures,” Remote Sens. Lett., vol. 5, no. 5, pp. 451-460, 2014.

[15] D. Capel, “An Effective Bail-out Test for RANSAC Consensus Scoring,” in Proc. Brit. Mach. Vis. Conf., 2005, pp. 629-638.

[16] R. Litman, S. Korman, and A. Bronstein, “Inverting RANSAC: Global Model Detection via Inlier Rate Estimation,” in Proc. IEEE Conf. Comput. Vis. Pattern Recognit., 2015, pp.5243-5251.

[17] H. Wang, J. Cai, J. Tang, “AMSAC: An adaptive robust estimator for model fitting," in Proc. IEEE Int. Conf. Image Processing., 2013, pp.305-309.

[18] K. Lebeda, J. Matas, and O. Chum, "Fixing the Locally Optimized RANSAC," in Proc. Brit. Mach. Vis. Conf., 2012, pp. 95.1-95.11.

[19] K. Ni, H. Jin and F. Dellaert, "GroupSAC: Efficient consensus in the presence of groupings," in Proc. Int. Conf. Comput. Vis., 2009, pp. 2193-2200. 
[20] S. Nagaraja, C.J. Prabhakar, and P.U. Praveen Kumar, "Parallax effect free mosaicing of underwater video sequence based on texture features," Signal and Image Proc., vol. 5, no. 5, pp. $13-25,2014$.

[21] R. Hess, “An open-source SIFTLibrary,” in Proc. Int. Conf. Mult., 2010, pp, 1493-1496.

[22] J.M. Keller, M.R. Gray, and J.A. Givens Jr., “A fuzzy K-nearest neighbor algorithm,” IEEE Trans. Syst. Man Cybern., vol. SMC-15, no. 4, pp. 580-585, 1985.

[23] Hubert W. Lilliefors, "On the Komogorov-Smirnov test for normality with mean and variance unknown,” J. Am. Stat. Assoc., vol. 62, no. 318, pp. 399-402, 1967.

[24] L. Magri and A. Fusiello, "T-Linkage: a Continuous Relaxation of J-Linkage for Multi-Model Fitting,” in Proc. IEEE Conf. Comput. Vis. Pattern Recognit., 2014, pp. 3954-3961.

[25] The ISPRS Dataset [Online]. Available: http://www.isprs. org/data/

[26] J. Philbin, O. Chum, M. Isard, J. Sivic, and A. Zisserman, "Object retrieval with large vocabularies and fast spatial matching," in Proc. IEEE Conf. Comput. Vis. Pattern Recognit., 2007, pp. 1-8 .

[27] V. Chandrasekhar, D. Chen, S. Tsai, N. Cheung, H. Chen, G. Takacs, Y. Reznik, R. Vedantham, R. Grzesczcuk, J. Bach, and B. Girod, "The Stanford Mobile Visual Search Data Set," in Proc. 2nd ACM Conf. Multimedia Syst., 2011, pp. 117-122.

[28] G. Yang, C.V. Stewart, M. Sofka, and C. Tsai, "Registration of Challenging Image Pairs: Initialization, Estimation, and Decision,” IEEE Trans. Pattern Anal. Mach. Intell., vol. 29, no. 11, pp. 1973-1989, 2007.

[29] K. Lebeda, J. Matas, and O. Chum, "Fixing the Locally Optimized RANSAC - Full Experimental Evaluation,” Czech Technical University, Prague, Czech Republic, Rep. CTU-CMP-2012-17, Sept.12, 2012. 


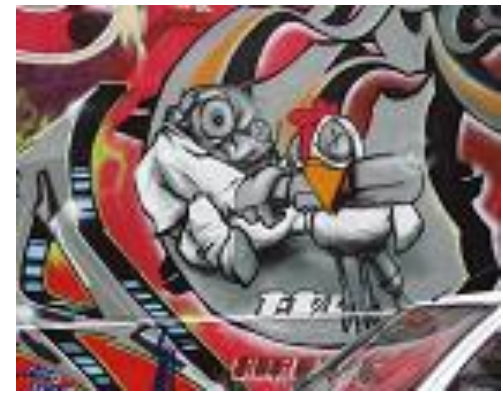

(a)

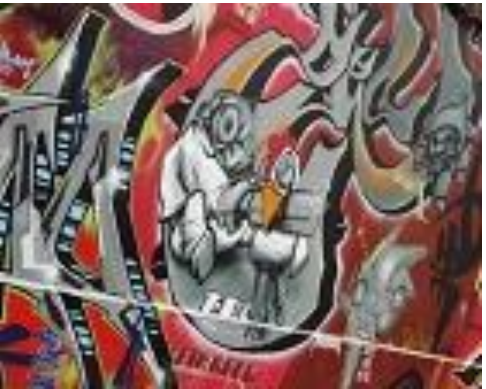

(b)

Fig. 1 Two "graffiti" images with different viewpoint.
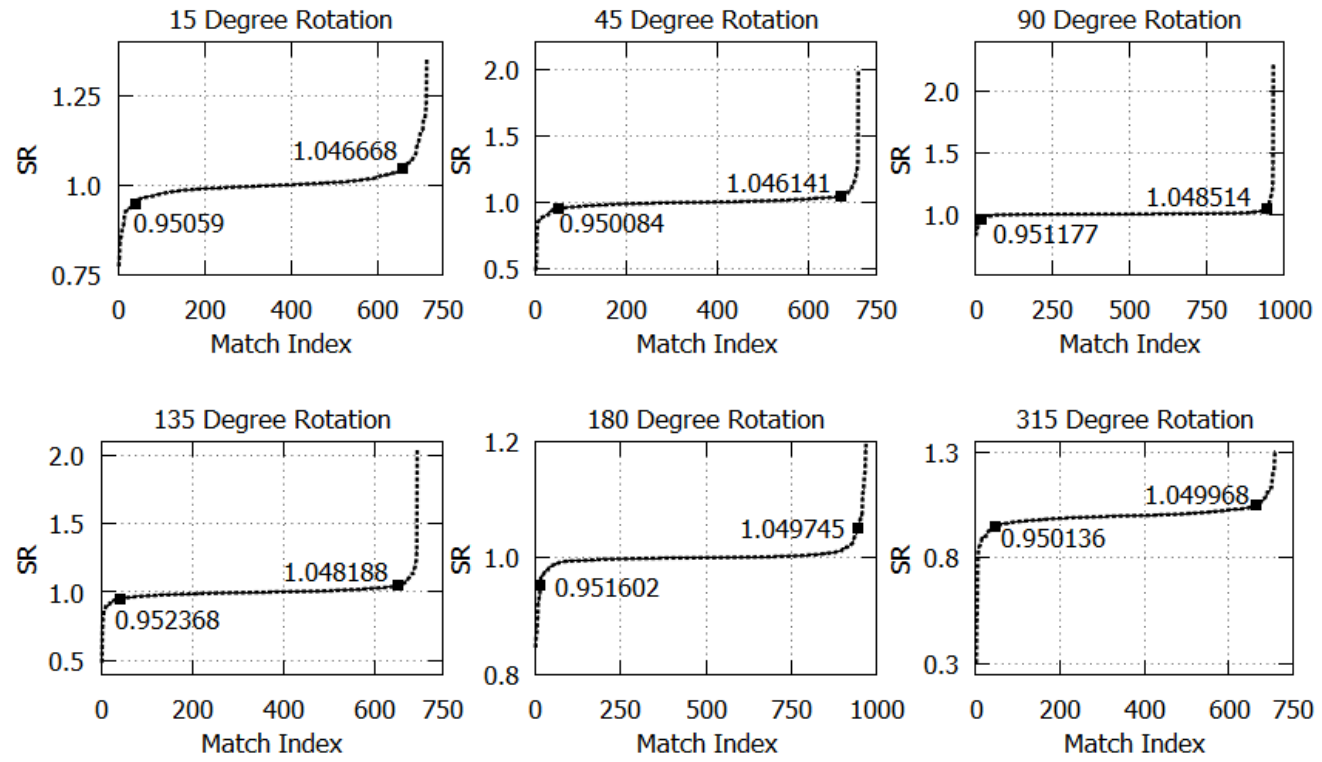

Fig. 2 The $S R$ distributions for different rotate transformation. For each graph, the index of matches is on the $\mathrm{x}$-axis and the corresponding $S R$ of each match is on the y-axis. Two numerical values on each $S R$ distribution curve are the real boundary values (inclusive) in the range [0.95,1.05].

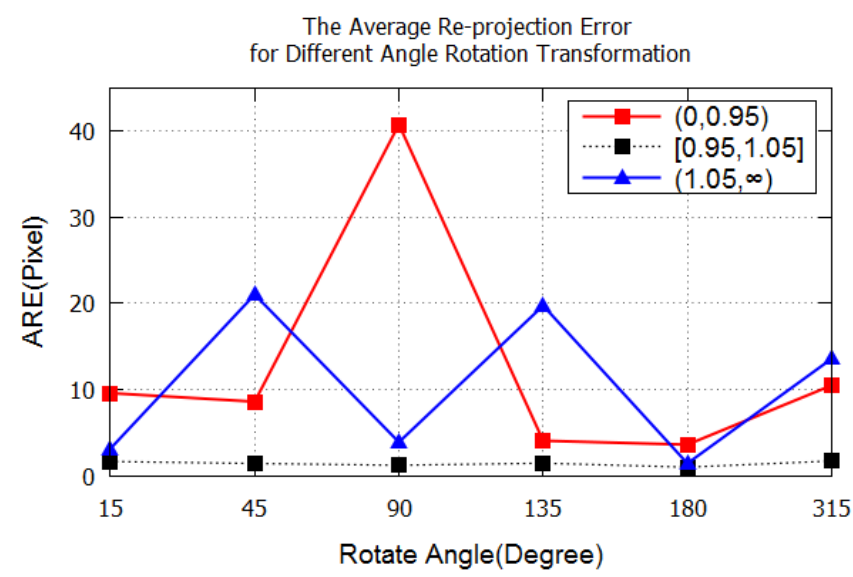

Fig. 3 The average re-projection error distribution for different rotate transformation. The rotate angle is on the $\mathrm{x}$-axis and the average re-projection error is on the $\mathrm{y}$-axis. 

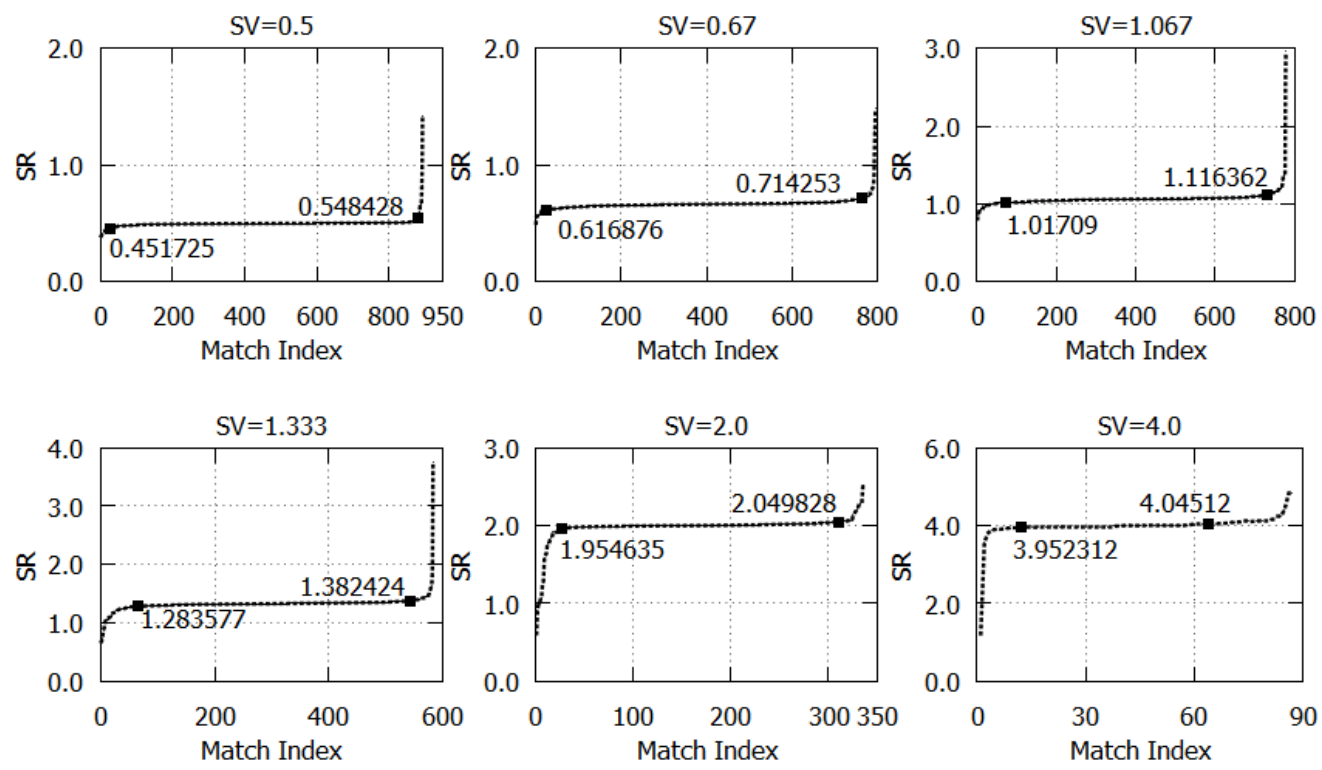

Fig. 4 The $S R$ distributions for different zoom transformation. On each graph, the index of matches is on the x-axis and the corresponding $S R$ of each match is on the y-axis. Two numerical values on each $S R$ distribution curve are the real boundary values (inclusive) in the range $[S V-0.05, S V+0.05]$.

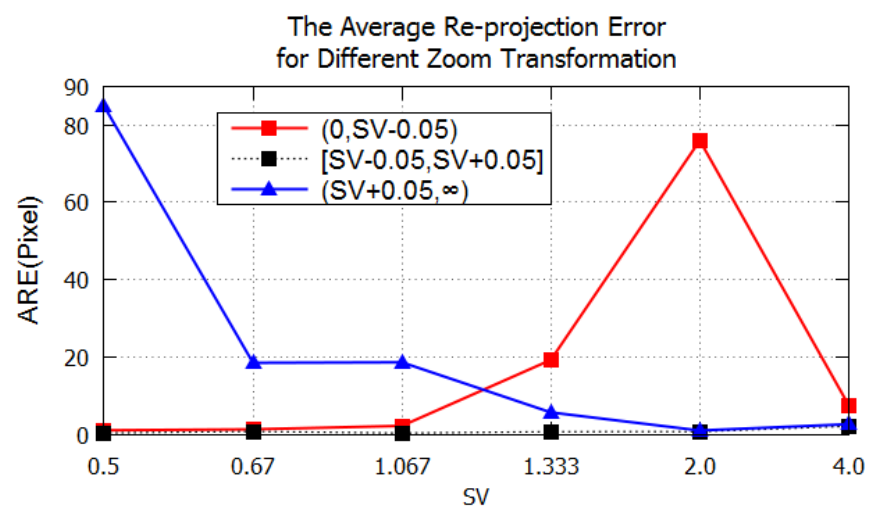

Fig. 5 The average re-projection error distribution for different zoom transformation. The $S V$ is on the $\mathrm{x}$-axis and the average re-projection error is on the y-axis.

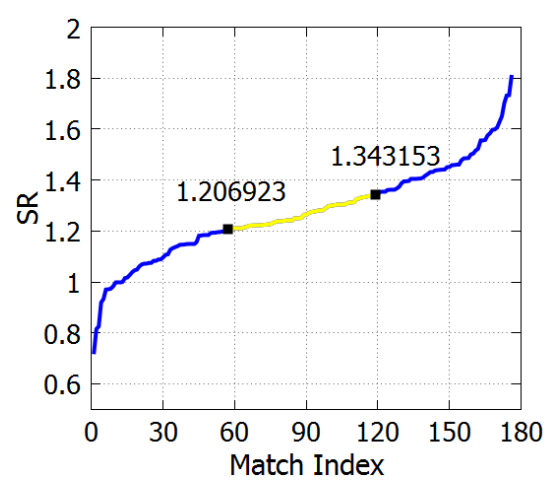

Fig. 6 The $S R$ distribution for two real images. The index of matches is on the $\mathrm{x}$-axis and the corresponding $S R$ of each match is on the y-axis. Two numerical values on the $S R$ distribution curve are the real boundary values (inclusive) in the range $[\overline{S R}-0.07, \overline{S R}+0.07]$. 


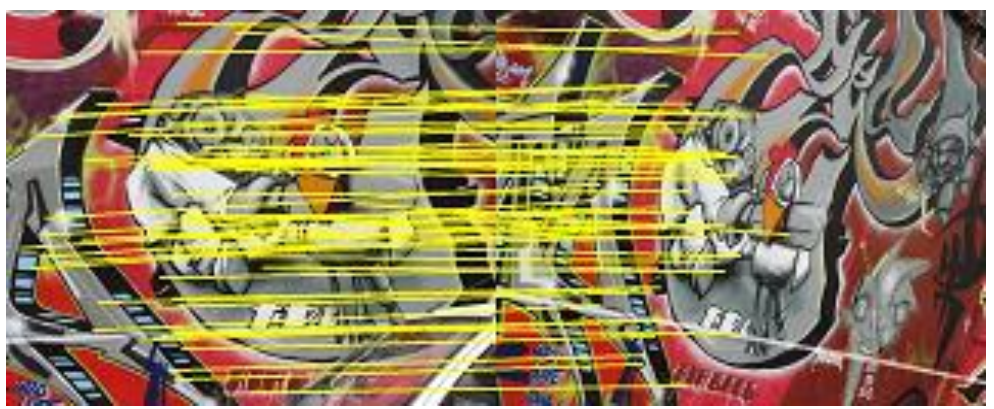

Fig. 7 The matches in $[\overline{S R}-0.07, \overline{S R}+0.07]$ depicted in yellow lines.

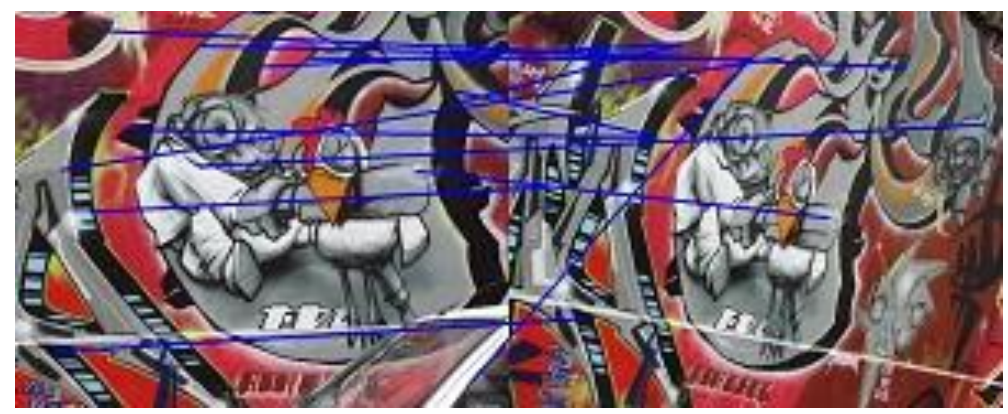

Fig. 8 The real false matches out of $[\overline{S R}-0.07, \overline{S R}+0.07]$ depicted in blue lines.

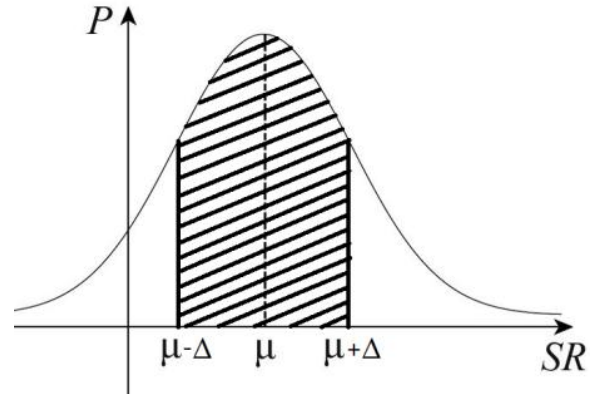

Fig. 9 The normal distribution of $S R$ with mean $\mu$.

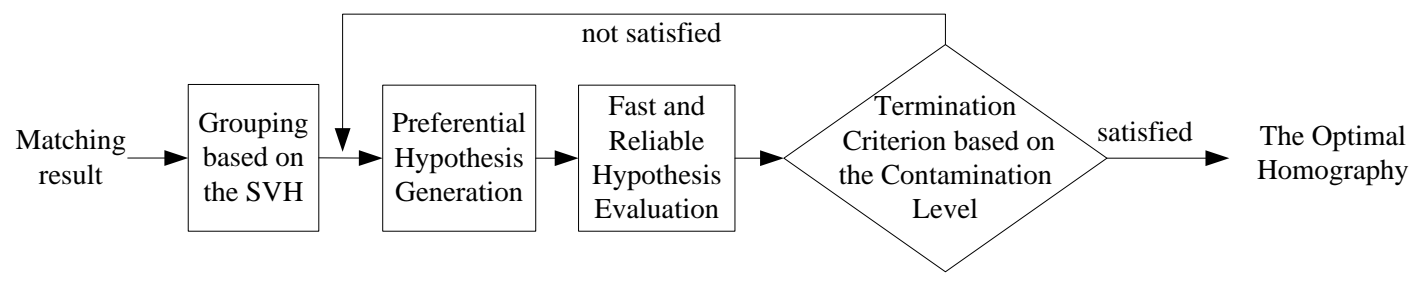

Fig. 10 The framework of SVH_RANSAC. 

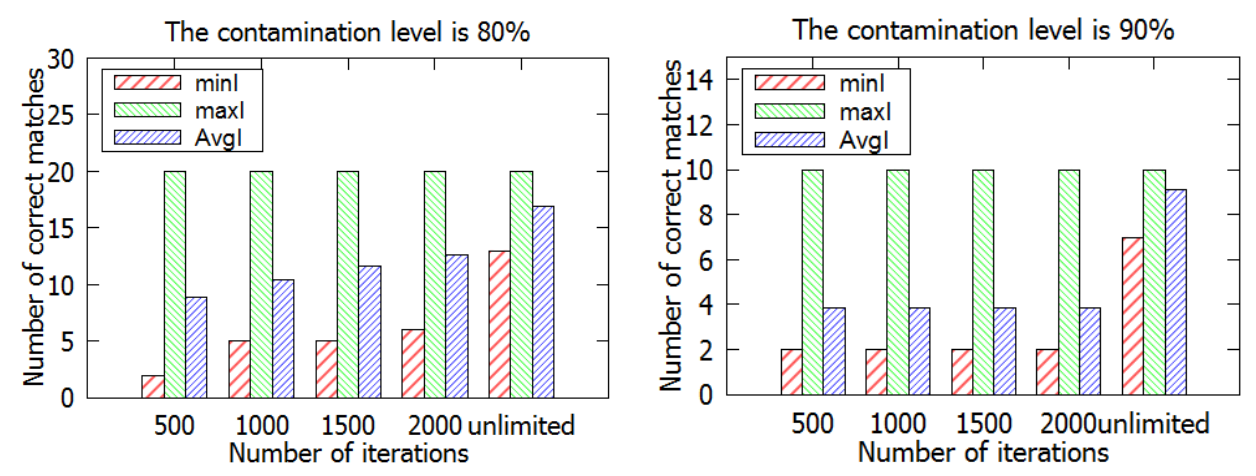

(a)RANSAC
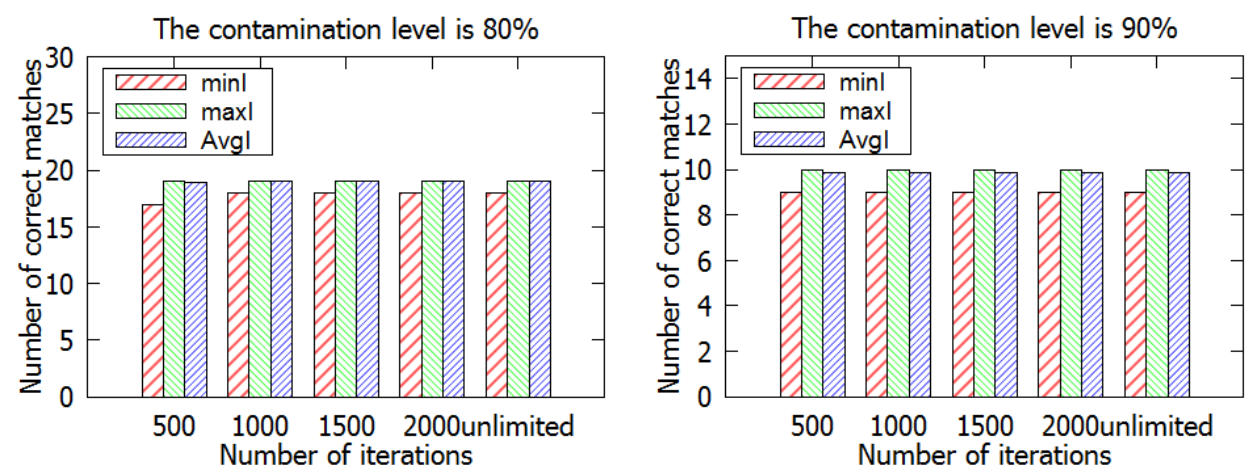

(b)PROSAC
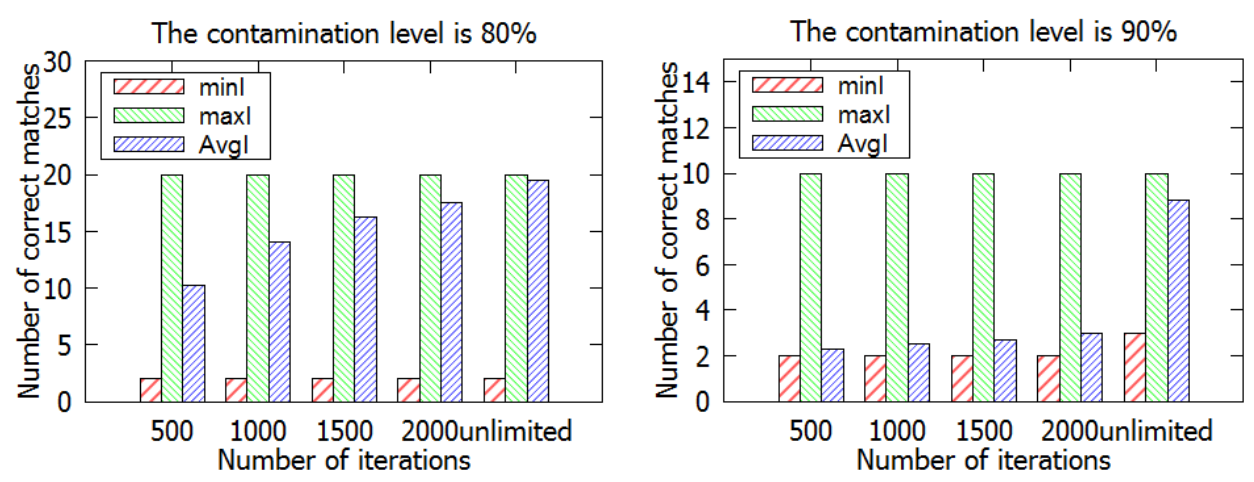

(c) $\mathrm{LO}^{+}$-RANSAC
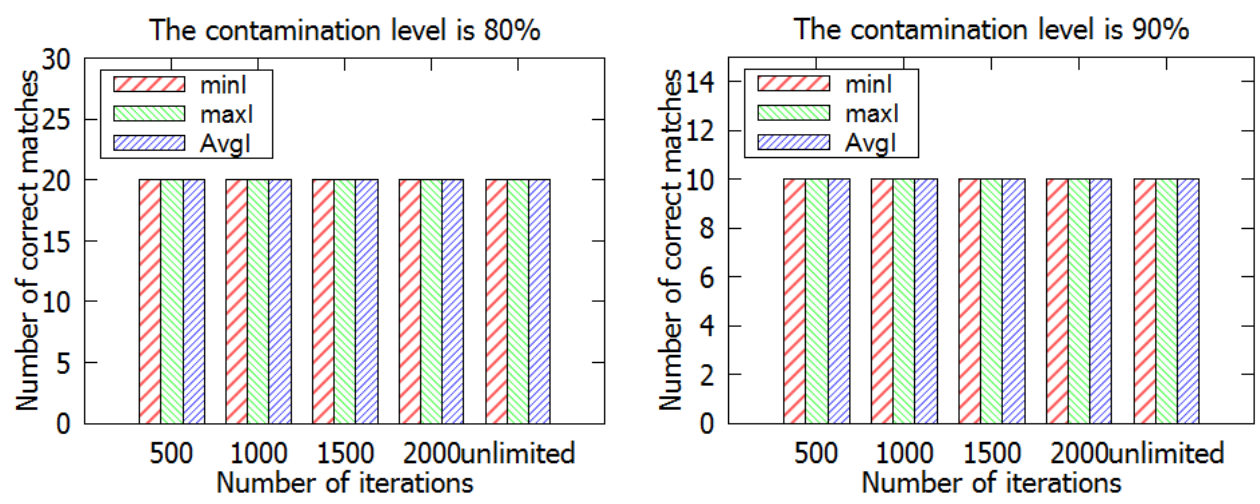

(d) SVH_RANSAC

Fig. 11 Comparison of the number of correct matches under different number of iterations. 


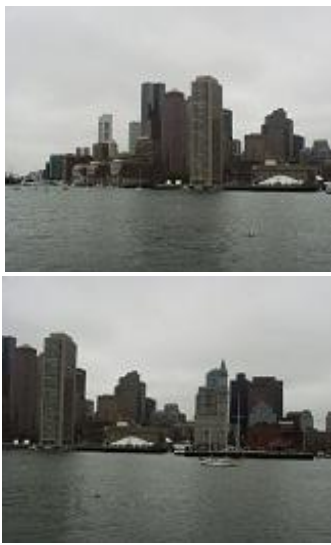

(a)Boston

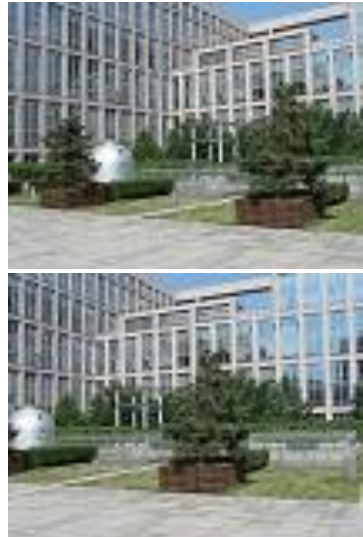

(d)Build

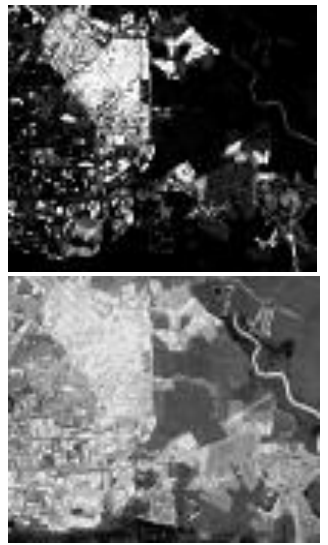

(b)City

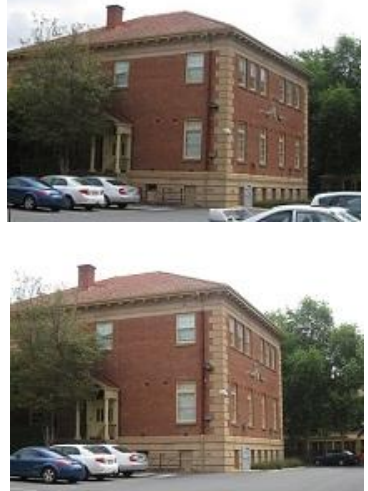

(c)Ladysymon

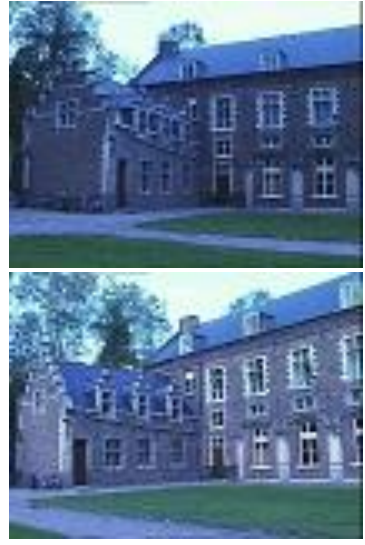

(e)Castle

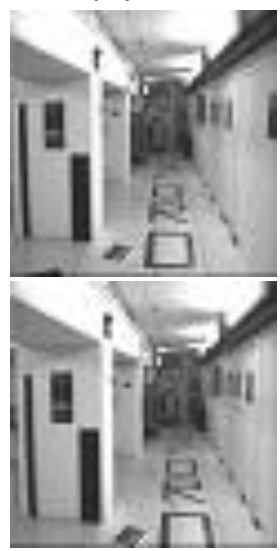

(f)Corr

Fig. 12 Six pairs of images with small parallax. 


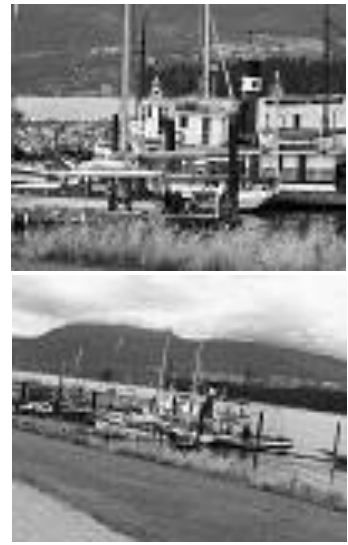

(a)Boat

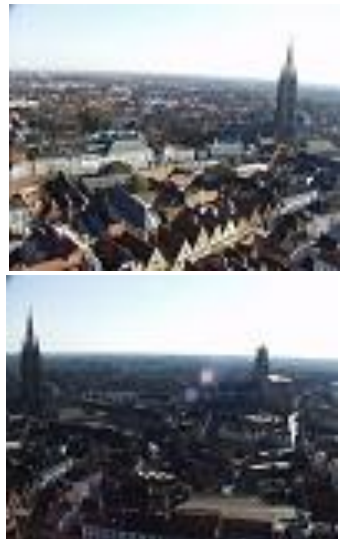

(d)BruggeTower

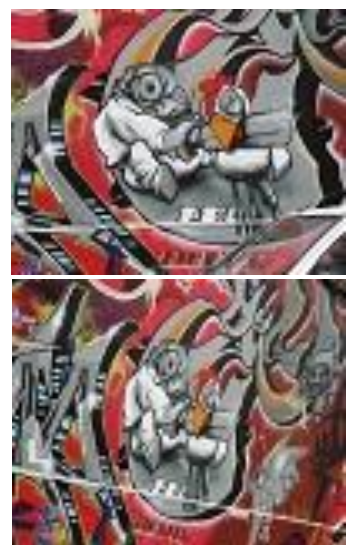

(g)Graffiti

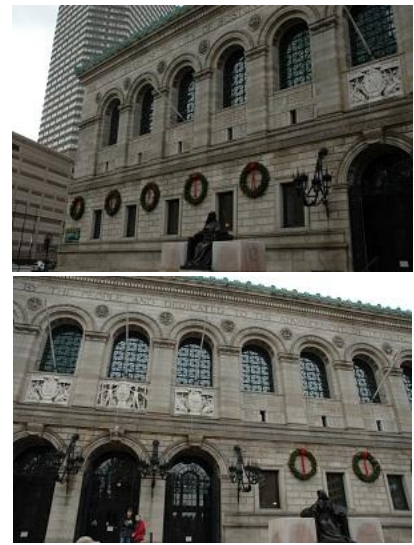

(b)BostonLib

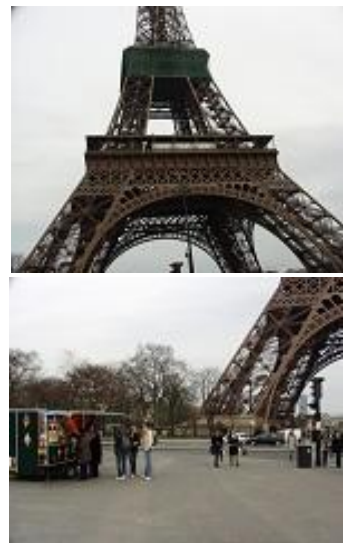

(e)Eiffel

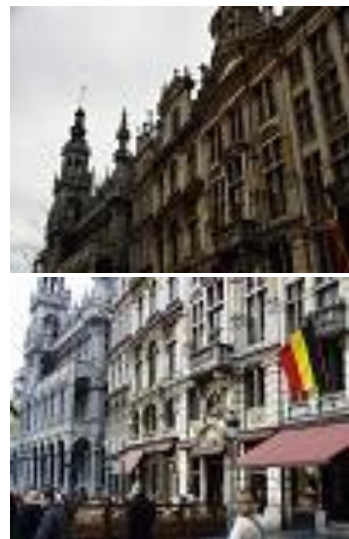

(h)Brussels

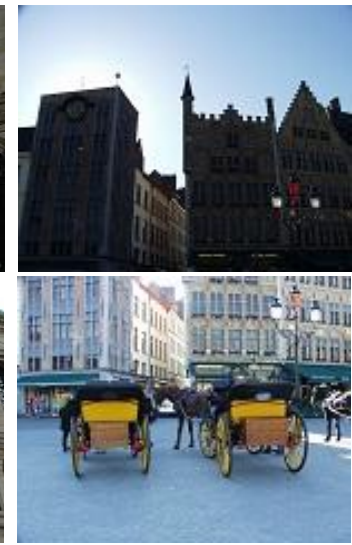

(c) BruggeSquare

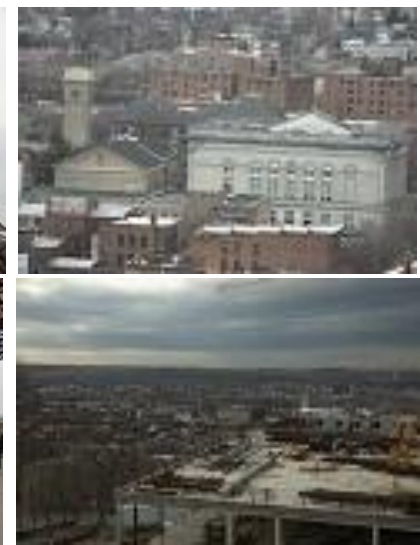

(f)ExtremeZoom

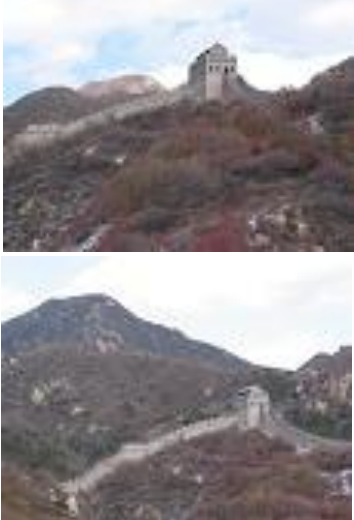

(i)GreatWall

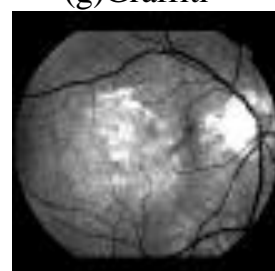

(j)Angiogram
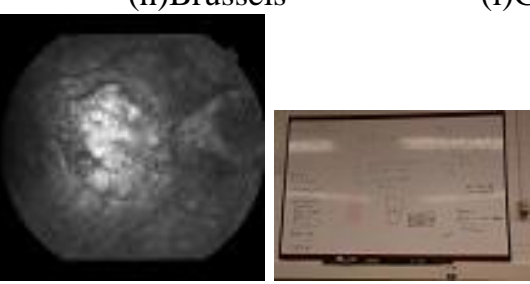

(k)WhiteBoard

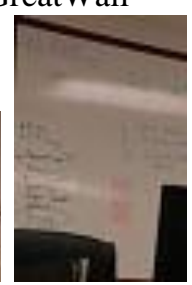

Fig. 13 Eleven pairs of images with large parallax. 


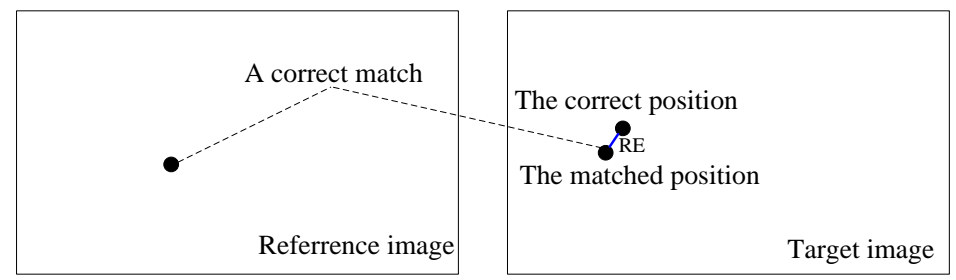

Fig. 14 The re-projection error of a correct match. 